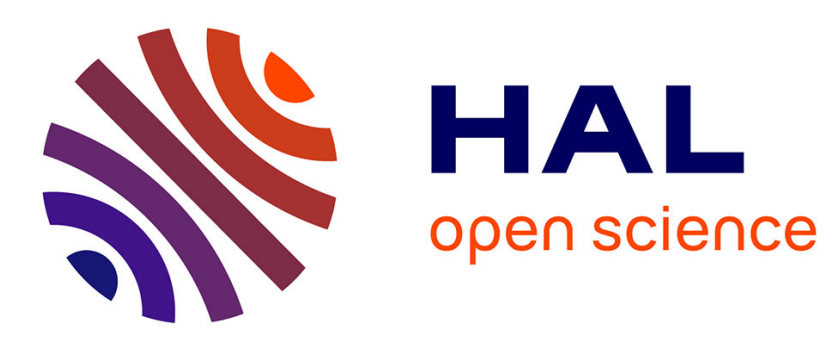

\title{
Internal wave generation in uniformly stratified fluids. Part 1. Green's function and point sources \\ Bruno Voisin
}

\section{To cite this version:}

Bruno Voisin. Internal wave generation in uniformly stratified fluids. Part 1. Green's function and point sources. Journal of Fluid Mechanics, 1991, 231, pp.439-480. 10.1017/S0022112091003464 . hal-01943687

\section{HAL Id: hal-01943687 \\ https://hal.science/hal-01943687}

Submitted on 8 Dec 2018

HAL is a multi-disciplinary open access archive for the deposit and dissemination of scientific research documents, whether they are published or not. The documents may come from teaching and research institutions in France or abroad, or from public or private research centers.
L'archive ouverte pluridisciplinaire HAL, est destinée au dépôt et à la diffusion de documents scientifiques de niveau recherche, publiés ou non, émanant des établissements d'enseignement et de recherche français ou étrangers, des laboratoires publics ou privés. 


\title{
Internal wave generation in uniformly stratified fluids. Part 1. Green's function and point sources
}

\author{
By BRUNO VOISIN
}

Thomson-Sintra Activités Sous-Marines, 1, avenue A. Briand, 94117 Arcueil, France and Département de Recherches Physiques, Université Pierre et Marie Curie, 4, place Jussieu, 75252 Paris, France

(Received 21 July 1990 and in revised form 4 April 1991)

In both Boussinesq and non-Boussinesq cases the Green's function of internal gravity waves is calculated, exactly for monochromatic waves and asymptotically for impulsive waves. From its differentiation the pressure and velocity fields generated by a point source are deduced. By the same method the Boussinesq monochromatic and impulsive waves radiated by a pulsating sphere are investigated.

Boussinesq monochromatic waves of frequency $\omega<N$ are confined between characteristic cones $\theta=\arccos (\omega / N)$ tangent to the source region ( $N$ being the buoyancy frequency and $\theta$ the observation angle from the vertical). In that zone the point source model is inadequate. For the sphere an explicit form is given for the waves, which describes their conical $1 / r^{\frac{1}{2}}$ radial decay and their transverse phase variations.

Impulsive waves comprise gravity and buoyancy waves, whose separation process is non-Boussinesq and follows the arrival of an Airy wave. As time $t$ elapses, inside the torus of vertical axis and horizontal radius $2 N t / \beta$ for gravity waves and inside the circumscribing cylinder for buoyancy waves, both components become Boussinesq and have wavelengths negligible compared with the scale height $2 / \beta$ of the stratification. Then, gravity waves are plane propagating waves of frequency $N \cos \theta$, and buoyancy waves are radial oscillations of the fluid at frequency $N$; for the latter, initially propagating waves comparable with gravity waves, the horizontal phase variations have vanished and the amplitude has become insignificant as the Boussinesq zone has been entered. In this zone, outside the torus of vertical axis and horizontal radius $N t a$, a sphere of radius $a \ll 2 / \beta$ is compact compared with the wavelength of the dominant gravity waves. Inside the torus gravity waves vanish by destructive interference. For the remaining buoyancy oscillations the sphere is compact outside the vertical cylinder circumscribing it, whereas the fluid is quiescent inside this cylinder.

\section{Introduction}

Internal gravity waves in density-stratified fluids, and the similar inertial waves in rotating fluids, markedly differ from the more classical acoustic or electromagnetic waves. They are anisotropic, and dispersive. In the monochromatic regime hyperbolic differential equations, rather than elliptic equations, govern their propagation. As a consequence even the classical Fermat's principle does not hold for them (Barcilon \& Bleistein 1969). 
The group velocity theory (e.g. Lighthill 1978, $\$ 4.4$; Tolstoy $1973, \S 2.4$ ) proved to be an ideal means of studying the changes such unusual properties induce on wave propagation. Thus, the knowledge of such phenomena as scattering (Barcilon \& Bleistein 1969; Baines 1971) and guiding (Brekhovskikh \& Goncharov 1985, §10.5) of internal or inertial waves has now reached a degree of refinement comparable with that for classical waves. Internal or inertial wave generation remains, on the other hand, less known. Lighthill $(\mathbf{1 9 6 0}, 1965,1967)$ has developed in Fourier space and time a theory for the generation of anisotropic dispersive waves, which is especially successful in dealing with internal waves (Lighthill 1978, $\S 4.8-4.12$ ). Its crux is, again, group velocity. As for any Fourier-transform-based approach, Lighthill's theory primarily applies to initial perturbations, and oscillating or uniformly moving sources.

In this paper we initiate the development of an alternative theory of internal wave generation, which we intend to be complementary to his in that the waves are investigated in real space and time. Greater attention can, accordingly, be paid to sources with arbitrary time dependence; examples are arbitrarily moving sources, which will be considered in a forthcoming paper. Our approach is based upon the Green's function method (Morse \& Feshbach 1953, ch. 7): the internal wave field is built as a superposition of elementary impulses, each of which is represented by the Green's function. Although the theories of acoustic (cf. Pierce 1981) and electromagnetic (cf. Jackson 1975) wave radiation largely rely upon Green's functions, no attempt has been made before to develop a similar theory of internal wave radiation, except in some recent Soviet works where it has been sketched (Miropol'skii 1978; Sekerzh-Zen'kovich 1982), or introduced to solve particular problems (Gorodtsov \& Teodorovich 1980, 1983).

As a preliminary step towards this aim, the present paper is devoted to the Green's function itself. The fluid is assumed unbounded and non-rotating, the buoyancy frequency constant and the internal waves three-dimensional. First a synthesis and a completion of previous scattered and sometimes contradictory results about the Green's function are given. From them criteria are secondly deduced for the validity of two approximations of crucial importance in internal wave theory: the pointsource model and the Boussinesq approximation. To achieve this aim the internal wave field of a point source is deduced from the Green's function, and it is compared with that of a pulsating sphere.

In $\S 2$ we review the existing literature about the Green's function of internal waves. Then we derive in $\S 3$ their equation, and discuss the radiation condition for them. The properties of plane internal waves are recalled in $\$ 4$ and applied to monochromatic and impulsive point sources, for the interpretation of results to follow. Section 5 describes the exact calculation of the monochromatic and impulsive Green's functions. An asymptotic evaluation of the latter, only obtained in $\$ 5$ in integral form, exhibits in $\$ 6$ the splitting of Boussinesq internal waves into gravity waves and buoyancy oscillations. The mechanism of the splitting is seen to be nonBoussinesq, and criteria are given for the validity of the Boussinesq approximation for each component. The analysis of gravity waves and buoyancy oscillations is continued in $\$ 7$, by deducing from the Green's function the pressure and velocity fields radiated by a point mass source. In $\$ 8$ a similar calculation of the Boussinesq internal waves generated by a pulsating sphere is made. Simultaneously the validity of equivalent point sources is examined, for either monochromatic or impulsive pulsations. 


\section{Bibliographical review}

Investigations of the Green's function of monochromatic internal waves generally involve Fourier transform methods. In this way Sarma \& Naidu $(1972 a, b)$, omitting the use of any radiation condition, then Ramachandra Rao $(1973,1975)$ and Tolstoy $(1973, \S 7.3)$, restoring it, derived non-Boussinesq internal waves generated by point mass and force sources. A similar calculation of the Boussinesq Green's function has been achieved by Rehm \& Radt (1975), and partially performed by Miles (1971), Sturova (1980) and Gorodtsov \& Teodorovich $(1980,1983)$ during studies of internal wave radiation by moving point sources. Unfortunately these results are often contradictory, like Ramachandra Rao's (1973, 1975) ones, which do not even coincide with each other.

A different angle of attack has more recently been adopted by some Soviet workers, who considered directly the Green's function of Boussinesq impulsive internal waves without resorting to any monochromatic intermediate step. Teodorovich \& Gorodtsov (1980) proved Miropol'skii's (1978) study by classical function theory to lead to erroneous conclusions, and Sekerzh-Zen'kovich's (1979, 1981) approach by generalized function theory to be the only valid one. The latter writer proposed both exact and asymptotic results. Zavol'skii \& Zaitsev (1984) then investigated the physical meaning, and applicability, of the asymptotic result. In so doing they but recovered an analysis by Dickinson (1969) who, as a particular case of acoustic-gravity waves, i.e. internal waves coupled with acoustic waves by compressibility, had performed a thorough asymptotic analysis of the Green's function of Boussinesq impulsive internal waves.

In a more general fashion a third series of studies have been devoted to the Green's function of acoustic-gravity waves and become, once the incompressible limit has been applied to them, relevant to non-Boussinesq internal waves. Pierce (1963), by a shrewd formulation of the radiation condition, Moore \& Spiegel (1964), by Lighthill's (1960) asymptotic method, and Grigor'ev \& Dokuchaev (1970), by the use of Fourier transforms, independently obtained three coinciding expressions of the monochromatic Green's function. Kato (1966a) made a more detailed examination of the equivalence of the first two derivations. He later (Kato 1966 b) deduced from the group velocity theory, as did Cole \& Greifinger (1969) from a stationary phase analysis, the wavefront structure of the impulsive Green's function. Then, Dickinson (1969) and Liu \& Yeh (1971) derived the asymptotic expansion of the associated waves while Row (1967) described a hydrostatic, that is low-frequency, approximation for them.

Experiments about the generation of internal waves have involved bodies of finite dimensions, and various shapes and motions. Monochromatic oscillations of a cylinder (Mowbray \& Rarity 1967; Kamachi \& Honji 1988) or a sphere (McLaren et al. 1973), impulsive oscillation of a cylinder (Stevenson 1973) and free motion of a displaced solid (Larsen 1969) or fluid (McLaren et al. 1973) sphere have all been investigated.

Few theoretical studies consider the generation of internal waves by sources of finite size from a general point of view. All of them are asymptotic and rely on farfield or large-time hypotheses. Under the Boussinesq approximation this problem has been solved by Lighthill $(1978, \S 4.10)$ and Chashechkin \& Makarov (1984) for extended monochromatic and transient mass sources, respectively, and Lighthill $(1978, \S 4.8)$ and Sekerzh-Zen'kovich (1982) for initial perturbations of the stratified medium. 
Most theoretical works deal with the motion of rigid bodies of specified shape. Bretherton (1967) and Grimshaw (1969) investigated the generation of Boussinesq internal waves by the impulsively started uniform motion of respectively a cylinder and a sphere. Hendershott (1969) similarly considered the impulsively started monochromatic pulsations of a sphere, and Larsen (1969) its free oscillations; Larsen however focused his attention on the oscillations themselves rather than the waves they produce. All subsequent works have been devoted to steady monochromatic internal waves, radiated by a cylinder (Appleby \& Crighton 1986), a sphere (Appleby \& Crighton 1987), a spheroid, either considered explicitly (Sarma \& Krishna 1972) or under the slender-body approximation (Krishna \& Sarma 1969), and a slender body of arbitrary axisymmetric shape (Rehm \& Radt 1975). Of the monochromatic studies only the latter two assume Boussinesq internal waves.

\section{Statement of the problem}

\subsection{Internal wave equation}

In an unbounded incompressible fluid with uniform stratification, that is where the undisturbed density $\rho_{0}$ varies exponentially with height $z$ according to

$$
\rho_{0}(z)=\rho_{00} \mathrm{e}^{-\beta z},
$$

the buoyancy (or Brunt-Väisälä) frequency $N=(g \beta)^{\frac{1}{2}}$ is constant. The smallamplitude internal waves generated by a mass source of strength $m$ per unit volume are described by the linearized equations of fluid dynamics (Brekhovskikh \& Goncharov 1985, 110.1 ; Lighthill 1978, §4.1):

$$
\begin{gathered}
\rho_{0} \frac{\partial \boldsymbol{v}}{\partial t}=-\boldsymbol{\nabla} P-\rho \mathrm{g} \boldsymbol{e}_{z}, \\
\boldsymbol{\nabla} \cdot \boldsymbol{v}=m \\
\frac{\partial \rho}{\partial t}=\rho_{0} \beta v_{z}
\end{gathered}
$$

Here $v, P$ and $\rho$ are respectively the velocity, pressure and density perturbations, and $e_{z}$ a unit vector along the $z$-axis directed vertically upwards. Subscripts $h$ and $z$ will hereafter denote horizontal and vertical components of vectors and operators.

Inferring from (3.2) that the motion is irrotational in the horizontal plane, we express $v_{\mathrm{h}}$ and $P$ in terms of a horizontal velocity potential $\phi$ (Miles 1971), eliminate $\rho$ using (3.4) and remark that the resulting system of equations for $v_{z}$ and $\phi$ is satisfied if

$$
\begin{gathered}
v=\left[\frac{\partial^{2}}{\partial t^{2}}\left(\nabla-\beta e_{z}\right)+N^{2} \nabla_{\mathrm{h}}\right] \psi \\
P=-\rho_{0}\left(\frac{\partial^{2}}{\partial t^{2}}+N^{2}\right) \frac{\partial}{\partial t} \psi \\
\rho=\rho_{0} \beta\left(\frac{\partial}{\partial z}-\beta\right) \frac{\partial}{\partial t} \psi
\end{gathered}
$$

with

$$
\left[\frac{\partial^{2}}{\partial t^{2}}\left(\nabla^{2}-\beta \frac{\partial}{\partial z}\right)+N^{2} \nabla_{\mathrm{h}}^{2}\right] \psi=m .
$$


$\nabla^{2}=\partial^{2} / \partial x^{2}+\partial^{2} / \partial y^{2}+\partial^{2} / \partial z^{2}$ and $\nabla_{h}^{2}=\partial^{2} / \partial x^{2}+\partial^{2} / \partial y^{2}$ are respectively the three- and two-dimensional Laplacians. The 'internal potential' $\psi$ generalizes to uniformly stratified fluids the velocity potential of homogeneous fluids, and simultaneously exhibits the creation of vorticity by the stratification. Its introduction dates back to Gorodtsov \& Teodorovich (1980) for Boussinesq internal waves, and Hart (1981) in a more general context.

The Boussinesq approximation consists of neglecting the inertial effects of density variations compared with the buoyancy forces they create. It is equivalent to setting $\beta \rightarrow 0$ and $g \rightarrow \infty$ with $g \beta=N^{2}$ held fixed in the preceding equations. Lighthill (1978, $\S 4.2)$ pointed out that, when non-Boussinesq effects are taken into account, the compressibility of the fluid should be also, transforming internal waves into acoustic-gravity waves. Studies of the generation of these waves (e.g. Liu \& Yeh 1971) showed them, however, to be made of the superposition of low-frequency nearly incompressible gravity waves and high-frequency acoustic waves slightly affected by gravity, constituting two distinct wavefronts with different propagation velocities. Therefore, non-Boussinesq incompressible internal waves have an existence of their own. As acoustic-gravity waves are approached, whose characteristics are the buoyancy frequency $N$, the sound velocity $c$ and the acoustic cutoff frequency $\omega_{\mathfrak{a}}$, incompressibility means the limiting process (cf. Tolstoy 1973, $\S 2.2) c \rightarrow \infty$ and $\omega_{\mathrm{a}} \rightarrow \infty$ with $\omega_{\mathrm{a}} / c \rightarrow \frac{1}{2} \beta$ and $N$ held fixed.

Removing from $v, P, \rho$ and $\psi$ density factors ensuring vertical conservation of energy, according to

$$
(\psi, v)=\mathrm{e}^{+\beta z / 2}\left(\psi^{\prime}, \boldsymbol{v}^{\prime}\right) \text { and }(P, \rho)=\mathrm{e}^{-\beta z / 2}\left(P^{\prime}, \rho^{\prime}\right),
$$

simplifies the internal wave equation (3.8) to

$$
\begin{gathered}
{\left[\frac{\partial^{2}}{\partial t^{2}}\left(\nabla^{2}-\frac{1}{4} \beta^{2}\right)+N^{2} \nabla_{\mathrm{h}}^{2}\right] \psi^{\prime}=\mathrm{e}^{-\beta z / 2} m,} \\
\boldsymbol{v}^{\prime}=\left[\frac{\partial^{2}}{\partial t^{2}}\left(\nabla-\frac{1}{2} \beta \boldsymbol{e}_{z}\right)+N^{2} \nabla_{\mathrm{h}}\right] \psi^{\prime}, \\
P^{\prime}=-\rho_{00}\left(\frac{\partial^{2}}{\partial t^{2}}+N^{2}\right) \frac{\partial}{\partial t} \psi^{\prime} \\
\rho^{\prime}=\rho_{00} \beta\left(\frac{\partial}{\partial z}-\frac{\beta}{2}\right) \frac{\partial}{\partial t} \psi^{\prime} .
\end{gathered}
$$

Hereafter $v^{\prime}, P^{\prime}, \rho^{\prime}$ and $\psi^{\prime}$ will implicitly be considered and primes will be omitted. While the extraction of density factors reduces non-Boussinesq effects upon the propagation of internal waves to $O\left(\beta^{2}\right)$ (Lighthill 1978, $\S 4.2$ ), there remain $O(\beta)$ effects upon their generation (Appleby \& Crighton 1986, 1987). This is exhibited by the non-Boussinesq term on the left-hand side of $(3.10)$, and the source term on its right-hand side.

\subsection{Green's function and radiation condition}

From the mathematical point of view, the Green's function $G(r, t)$ of internal waves is defined as the Green's function of their operator, by

$$
\left[\frac{\partial^{2}}{\partial t^{2}}\left(\nabla^{2}-\frac{1}{4} \beta^{2}\right)+N^{2} \nabla_{\mathrm{h}}^{2}\right] G(\boldsymbol{r}, t)=\delta(\boldsymbol{r}) \delta(t) .
$$

It physically represents the internal potential generated by an impulsive point mass source releasing a unit volume of fluid, from which the corresponding velocity, 
pressure and density fields are deduced by (3.11)-(3.13). Accordingly, we shall refer to it as an impulsive Green's function and also introduce a monochromatic Green's function $G(\boldsymbol{r}, \omega)$, as the internal potential generated by a monochromatic point source, so that

$$
\left[\left(\omega^{2}-N^{2}\right) \nabla_{\mathrm{h}}^{2}+\omega^{2}\left(\frac{\partial^{2}}{\partial z^{2}}-\frac{\beta^{2}}{4}\right)\right] G(\boldsymbol{r}, \omega)=-\delta(\boldsymbol{r}) .
$$

They are related to each other by Fourier transformation in time, according to

$$
\begin{gathered}
G(\boldsymbol{r}, \omega)=\int G(\boldsymbol{r}, t) \mathrm{e}^{-\mathrm{i} \omega t} \mathrm{~d} t \equiv \mathrm{FT}[G(\boldsymbol{r}, t)], \\
G(\boldsymbol{r}, t)=\frac{1}{2 \pi} \int G(\boldsymbol{r}, \omega) \mathrm{e}^{\mathrm{i} \omega t} \mathrm{~d} \omega \equiv \mathrm{FT}^{-1}[G(\boldsymbol{r}, \omega)] .
\end{gathered}
$$

In fact, neither (3.14) nor (3.15) enables the complete determination of the Green's function, in that their solutions contain arbitrary linear combinations of free waves (corresponding to zero source terms). Similarly the presence, in inversion formula $(3.16 b)$, of singularities of the integrand over the real axis makes the evaluation of the integral ambiguous. In both cases a radiation condition must be added, which states that all waves originate at the source, none 'coming in from infinity' or having been generated before the source has begun to emit. So far, three different forms of this condition have been used to deal with internal wave generation: (i) Sommerfeld's radiation condition, (ii) Lighthill's radiation condition and (iii) Pierce's and Hurley's radiation condition.

Sommerfeld's radiation condition operates in the space domain, and expresses that all waves must be outgoing far from the source region. Its analytical form remains, for waves with fixed propagation velocity, simple (cf. Pierce 1981, §4.5). For internal waves it must, however, be replaced by the requirement that the group velocity point outward, a requirement which is written differently for each plane wave composing their spectrum. Under the geometrical approximation waves are locally plane and this condition takes on a simple form again (Barcilon \& Bleistein 1969). When diffraction effects take place the whole spectrum must be taken into account and the radiation condition must be written, either individually for each plane wave to be later superposed (Ramachandra Rao 1973), or simultaneously for all plane waves in which case unwieldy integral equations are involved (Baines 1971).

The radiation condition is more conveniently expressed for internal waves in the time domain as the principle of causality : no waves can be radiated before the source has been 'switched on'. Lighthill, Pierce and Hurley proposed different ways to apply causality to steady monochromatic waves. Lighthill $(1960,1965,1967,1978$, $\S 4.9)$ adds to the frequency $\omega$ a small negative imaginary part $-\epsilon$ which he later allows to tend to zero; he interprets this process as a gradual exponential growth of the source from its switching on at $t=-\infty$ to its present state. Pierce (1963), and independently Hurley (1972), consider a source abruptly switched on at $t=0$; they require that time Fourier transforms such as $G(\boldsymbol{r}, \omega)$ be analytic in the lower half of the complex $\omega$-plane and tend to zero as $\operatorname{Im} \omega \rightarrow-\infty$, so as to ensure that $G(r, t)$ is zero for negative times.

According to the causal radiation conditions the integration path in $(3.16 b)$ must lie below the real singularities, which we write as

$$
G(\boldsymbol{r}, t)=\frac{1}{2 \pi} \psi G(\boldsymbol{r}, \omega) \mathrm{e}^{\mathrm{i} \omega t} \mathrm{~d} \omega \equiv \mathrm{FT}^{-1}[G(\boldsymbol{r}, \omega)]
$$


This definition is described by Morse \& Feshbach (1953, pp. 460-461) as the natural extension of Fourier analysis to non-square-integrable functions, whose Fourier transforms only exist in a generalized function sense. It is not to be confused with the principal value of integral $(3.16 b)$, which does not verify causality. It is on the other hand equivalent to the Bromwich contour integral for Laplace transforms (cf. Appendix D).

\section{Plane internal waves}

Numerous writers have investigated the properties of Boussinesq (e.g. Lighthill 1978, §\$4.1 and 4.4; Brekhovskikh \& Goncharov 1985, §10.4) and non-Boussinesq (e.g. Tolstoy 1973, §2.4; Liu \& Yeh 1971) plane internal waves and have analysed, via the group velocity theory, the generation of internal waves by point sources. We summarize here their conclusions, for the interpretation of forthcoming results.

\subsection{Boussinesq case}

The dispersion relation for plane monochromatic Boussinesq internal waves $(\boldsymbol{k}$ is the wavevector, $k_{\mathrm{h}}$ its horizontal projection, and $k$ and $k_{\mathrm{h}}$ their moduli),

$$
\omega=N \frac{k_{\mathrm{h}}}{k},
$$

implies a frequency $\omega<N$, an arbitrary wavelength $\lambda$ and an inclination of the planes of constant phase to the vertical of $\theta_{0}=\arccos (\omega / N)$. The phase velocity $c_{\phi}$ with which these planes move and the group velocity $c_{\mathrm{g}}$ with which energy propagates are perpendicular, according to

$$
\begin{gathered}
c_{\phi}=\frac{\omega}{k} \frac{k}{k} \quad \text { with } \quad c_{\phi}=\frac{\omega}{k}=\frac{N}{k} \frac{k_{\mathrm{h}}}{k}=\frac{N}{k} \cos \theta_{0}, \\
c_{\mathrm{g}}=\frac{N}{k} \frac{k_{z}}{k_{\mathrm{h}}} \frac{k}{k} \times\left(\frac{k}{k} \times e_{z}\right) \quad \text { with } \quad c_{\mathrm{g}}=\frac{\left(N^{2}-\omega^{2}\right)^{\frac{1}{2}}}{k}=\frac{N}{k} \frac{\left|k_{z}\right|}{k}=\frac{N}{k} \sin \theta_{0} .
\end{gathered}
$$

Thus, energy propagates along the planes of constant phase. Conversely $k$ satisfies

$$
\boldsymbol{k}=\frac{N}{c_{\mathrm{g}}} \frac{c_{\mathrm{g}}}{c_{\mathrm{g}}} \times\left(\frac{c_{\mathrm{g}}}{c_{\mathrm{g}}} \times e_{\mathrm{z}}\right) \operatorname{sgn} c_{\mathrm{g}_{\mathrm{z}}} .
$$

By virtue of

$$
v=\frac{P}{\rho_{0} c_{\mathrm{g}}} \frac{c_{\mathrm{g}}}{c_{\mathrm{g}}},
$$

fluid particles move along straight-line paths also parallel to the wavecrests.

A monochromatic point source consequently radiates Boussinesq internal waves along directions inclined at the angle $\theta_{0}=\arccos (\omega / N)$ to the vertical, on a 'St Andrew's Cross' in two dimensions and a cone with vertical axis, hereafter called characteristic, in three dimensions (figure 1). Surfaces of constant phase are parallel to this cone. They move towards the level of the source, but disappear as soon as they have left the cone within which all the energy is confined. The motion of fluid particles is radial and also located on the cone. A confirmation of these features comes from the experiments of Mowbray \& Rarity (1967), McLaren et al. (1973) and Kamachi \& Honji (1988). Of particular importance are internal waves of nearbuoyancy frequency. They are confined at the vertical from the source and do not 


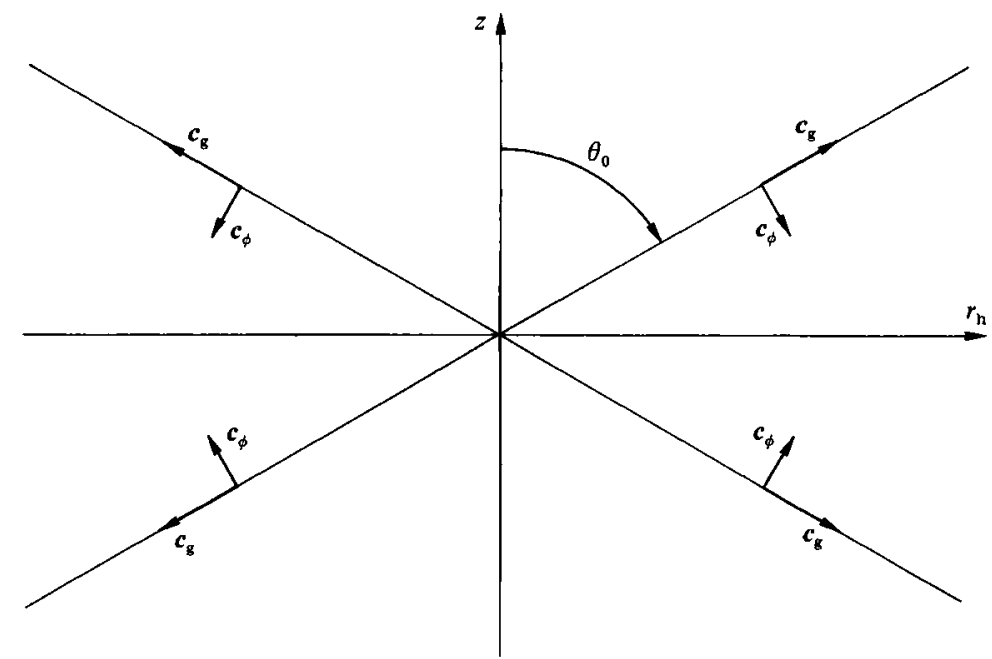

Figure 1. Boussinesq internal waves radiated by a monochromatic point source oscillating at frequency $\omega=\frac{1}{2} N$. Waves are confined on the characteristic cone of semi-angle $\theta_{0}=\arccos (\omega / N)$, along which energy propagates with group velocity $c_{\mathrm{g}}$. Surfaces of constant phase are parallel to the cone, and move perpendicular to it with phase velocity $c_{\phi}$.

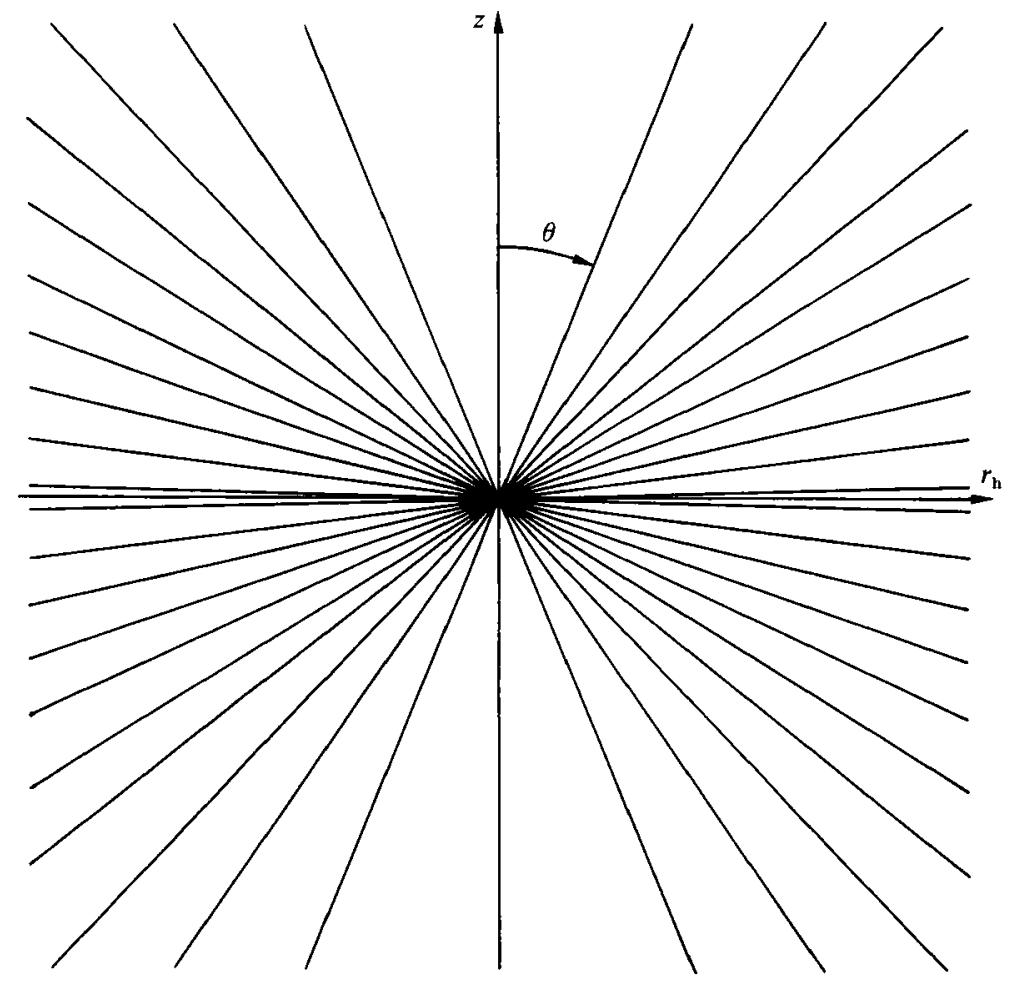

Fiaure 2. Boussinesq internal waves radiated by an impulsive point source. The conical surfaces of constant phase $\Phi=N t|\cos \theta|=\frac{1}{4} \pi+n \pi$ ( $n$ any integer), on which the pressure and velocity fields are zero, are shown for $N t=10 \pi$. 


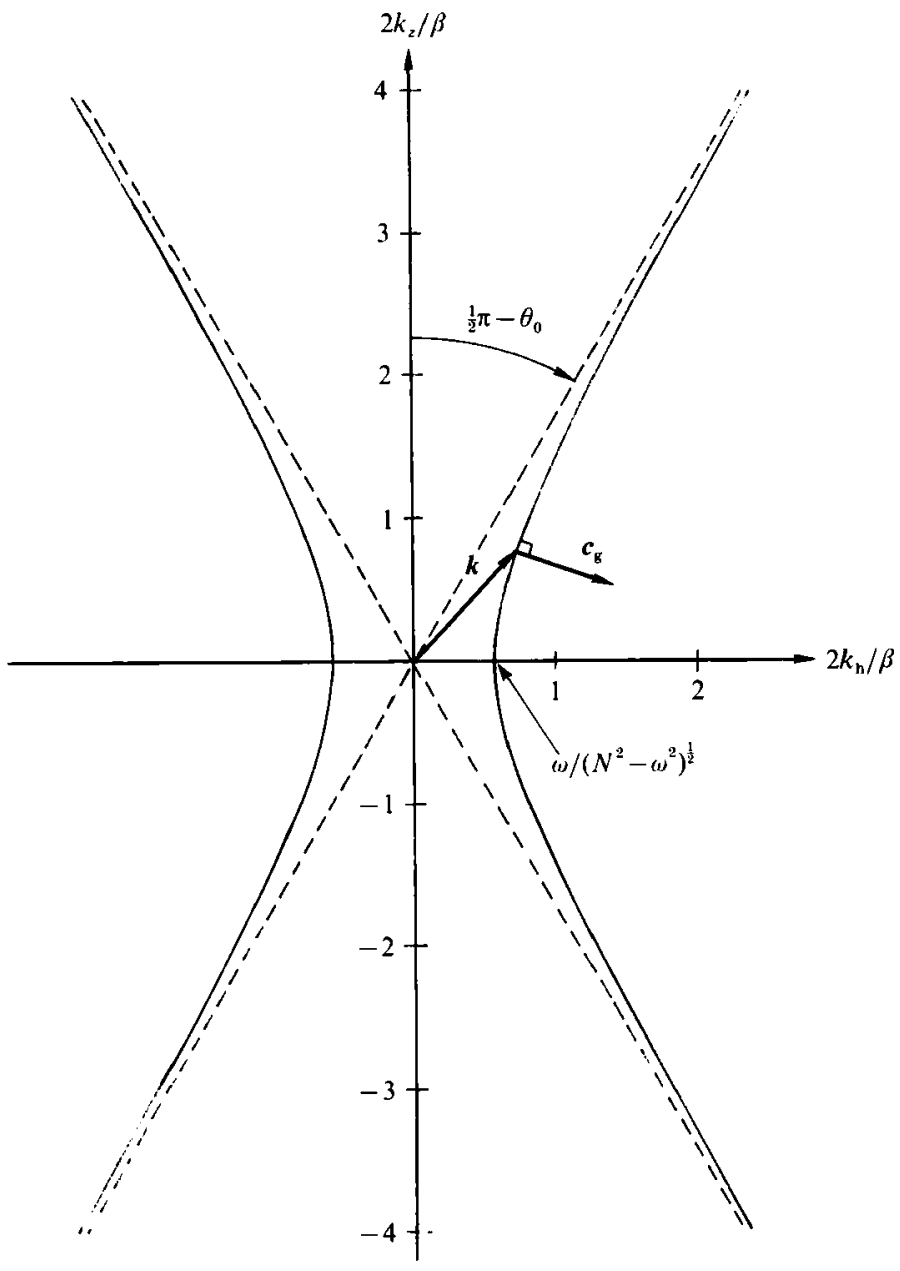

Figure 3. Wavenumber surface for non-Boussinesq internal waves of frequency $\omega=\frac{1}{2} N$. The group velocity $c_{\mathrm{g}}$ associated to a given wavevector $\boldsymbol{k}$ points along the normal to this surface, out of the characteristic cone of semi-angle $\theta_{0}=\arccos (\omega / N)$.

propagate, for their group velocity vanishes. They are instead vertical oscillations of fluid particles. This too is confirmed by the experiments of Gordon \& Stevenson (1972).

The Boussinesq internal waves generated by a point impulsive source propagate away from it at the group velocity $c_{\mathrm{g}}=r / t$, where $r$ represents the position with respect to the source and $t$ the time elapsed since the impulse. The frequency and wavevector in a direction inclined at an angle $\theta$ to the upward vertical follow from (4.4) and (4.1), as

$$
\omega=N|\cos \theta| \quad \text { and } \quad k=\frac{N t}{r} \frac{\boldsymbol{r}}{r} \times\left(\frac{\boldsymbol{r}}{r} \times e_{z}\right) \operatorname{sgn} z .
$$

The surfaces of constant phase $\Phi=\omega t-k \cdot r=N t|\cos \theta|$ are conical (figure 2) and move towards the level of the source at the decreasing phase speed

$$
c_{\phi}=\frac{r}{t}|\operatorname{cotan} \theta|
$$




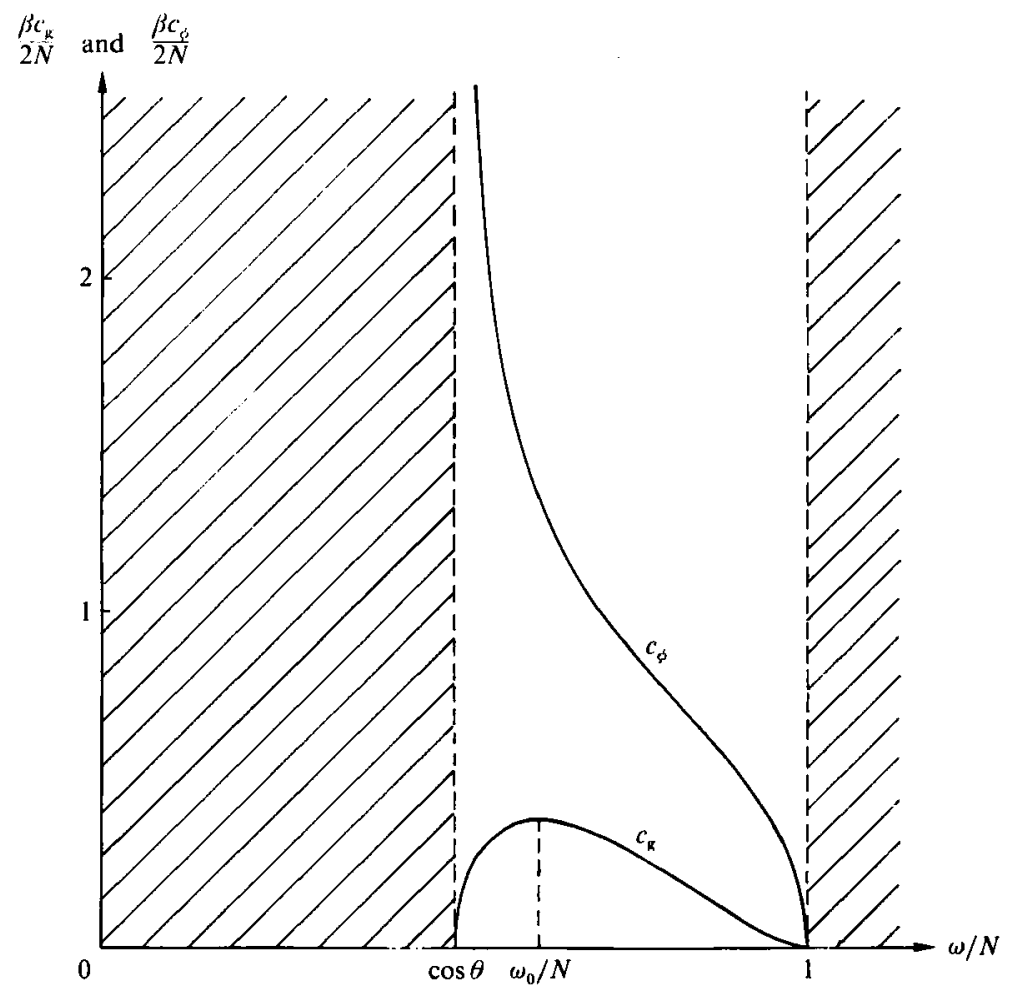

Fraure 4. Group velocity $c_{\mathrm{g}}$ and radial phase velocity $c_{\phi}$ of non-Boussinesq internal waves generated by a monochromatic point source as a function of frequency $\omega$, in a direction making an angle $\theta=60^{\circ}$ with the vertical.

in agreement with experiments by Stevenson (1973). The wavelength

$$
\lambda=\frac{2 \pi}{k}=\frac{2 \pi}{N t} \frac{r}{\sin \theta}
$$

is constant on toroidal surfaces with vertical axes and radii $N t \lambda / 2 \pi$. It decays with time, as the wavecrests multiply. Again, the motion of fluid particles is radial.

\subsection{Non-Boussinesq case}

Non-Boussinesq effects transform the dispersion relation into

$$
\omega=N \frac{k_{\mathrm{h}}}{\left(k^{2}+\frac{1}{4} \beta^{2}\right)^{\frac{1}{2}}} .
$$

The wavenumber surface is no longer a cone but an hyperboloidal surface of revolution, with asymptotes making an angle $\frac{1}{2} \pi-\theta_{0}$ with the vertical (figure 3 ). The group velocity points along its normal and is given by

$$
c_{\mathrm{g}}=N \frac{k^{2}}{\left(k^{2}+\frac{1}{4} \beta^{2}\right)^{\frac{3}{2}}} \frac{k_{z}}{k_{\mathrm{h}}} \frac{k}{k} \times\left(\frac{k}{k} \times e_{z}\right)+N \frac{\frac{1}{4} \beta^{2}}{\left(k^{2}+\frac{1}{4} \beta^{2}\right)^{\frac{3}{2}}} \frac{k_{\mathrm{h}}}{k_{\mathrm{h}}},
$$

or equivalently

$$
c_{\mathrm{g}_{\mathrm{h}}}=\frac{N^{2}-\omega^{2}}{\omega} \frac{\boldsymbol{k}_{\mathrm{h}}}{k^{2}+\frac{1}{4} \beta^{2}}, \quad c_{\mathrm{g}_{z}}=-\omega \frac{k_{z}}{k^{2}+\frac{1}{4} \beta^{2}} .
$$


It is no longer perpendicular to the wavevector. The trajectories of fluid particles become ellipses, studied by McLaren et al. (1973).

A point monochromatic source consequently radiates non-Boussinesq internal waves into the whole region $|\cos \theta|<\omega / N$ situated outside the characteristic cone. There, each point receives a wave whose group velocity is directed towards it and whose wavevector satisfies, according to (4.11),

$$
\begin{gathered}
\boldsymbol{k}_{\mathrm{h}}=\frac{\beta}{2} \frac{\omega^{2}}{\left[\left(N^{2}-\omega^{2}\right)\left(\omega^{2}-N^{2} \cos ^{2} \theta\right)\right]^{\frac{1}{2}}} \sin \theta \frac{r_{\mathrm{h}}}{r_{\mathrm{h}}}, \\
k_{z}=-\frac{\beta}{2}\left(\frac{N^{2}-\omega^{2}}{\omega^{2}-N^{2} \cos ^{2} \theta}\right)^{\frac{1}{2}} \cos \theta .
\end{gathered}
$$

The associated phase is of the form

$$
\Phi=\omega t-k \cdot r=\omega t-\frac{\beta r}{2}\left(\frac{\omega^{2}-N^{2} \cos ^{2} \theta}{N^{2}-\omega^{2}}\right)^{\frac{1}{2}} \equiv \omega t-\xi(\omega) r .
$$

Waves are locally plane, with a radial wavenumber $\xi(\omega)$, a radial phase velocity $\omega / \xi(\omega)$, and a group velocity

$$
c_{\mathrm{g}}=\frac{1}{\xi^{\prime}(\omega)}=\frac{2}{\beta} \frac{\left(N^{2}-\omega^{2}\right)^{\frac{3}{2}}\left(\omega^{2}-N^{2} \cos ^{2} \theta\right)^{\frac{1}{2}}}{N^{2} \omega \sin ^{2} \theta},
$$

which vanishes at the limits $N|\cos \theta|$ and $N$ of the internal wave spectrum and reaches a maximum $c_{\mathrm{g} 0}$ at the frequency (figure 4 )

$$
\omega_{0}=N\left[|\cos \theta| \frac{|\cos \theta|+\left(3+\cos ^{2} \theta\right)^{\frac{1}{2}}}{3}\right]^{\frac{1}{2}} .
$$

\section{Exact Green's function}

\subsection{Monochromatic Green's function}

For $\omega>N$, rescaling the coordinates according to

$$
r_{\mathrm{h}}^{\prime}=\frac{\omega}{N} \boldsymbol{r}_{\mathrm{h}}, \quad z^{\prime}=\frac{\left(\omega^{2}-N^{2}\right)^{\frac{1}{2}}}{N} z
$$

reduces equation (3.15) for the monochromatic Green's function $G(r, \omega)$ to a Helmholtz-like equation, whose Green's function is given for instance by Bleistein $(1984$, p. 177). Returning to the original coordinate system described in figure 5 yields

$$
G(r, \omega)=\frac{1}{4 \pi r} \frac{\exp \left[-\frac{\beta r}{2}\left(\frac{\omega^{2}-N^{2} \cos ^{2} \theta}{\omega^{2}-N^{2}}\right)^{\frac{1}{2}}\right]}{\left(\omega^{2}-N^{2}\right)^{\frac{1}{2}}\left(\omega^{2}-N^{2} \cos ^{2} \theta\right)^{\frac{1}{2}}} .
$$

As implied by the Pierce-Hurley radiation condition, the analytic continuation of this result over the lower half of the complex $\omega$-plane provides the value of the Green's function on the whole real $\omega$-axis. The branch cuts emanating from the 


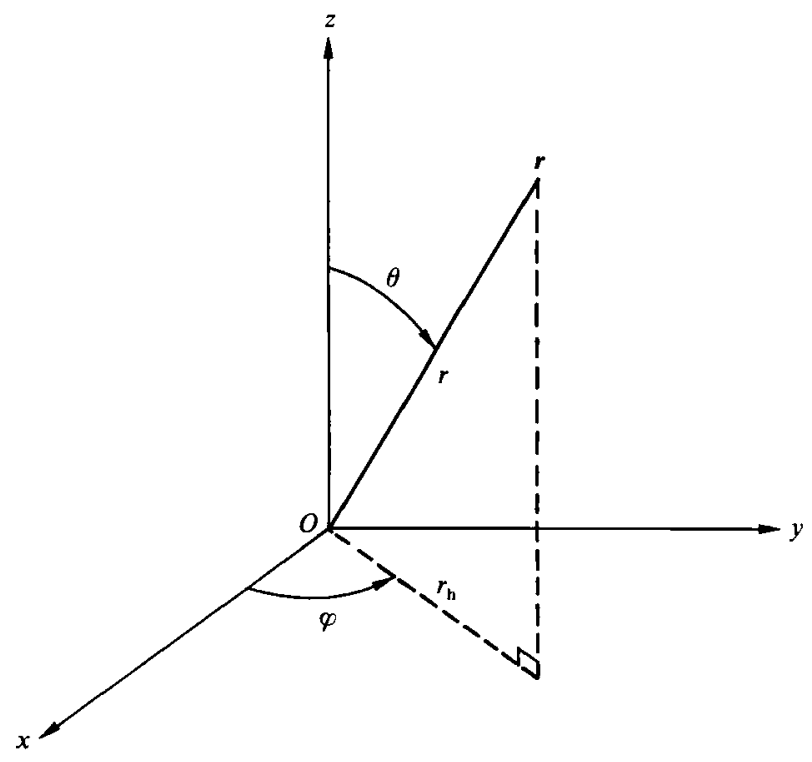

Figure 5. Coordinate system for internal wave radiation.

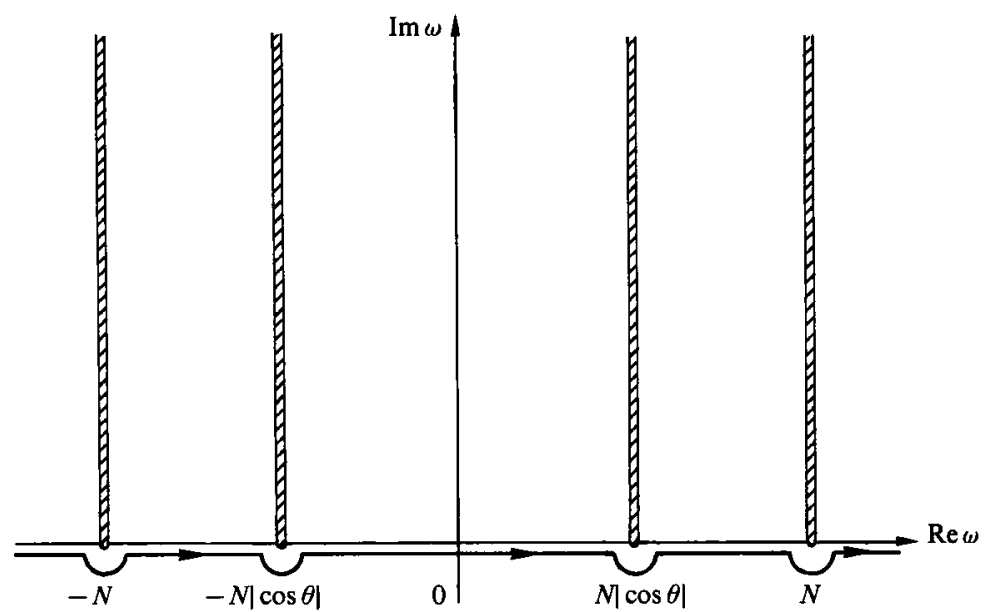

Figure 6. Branch cuts for the monochromatic Green's function, and integration path for the impulsive Green's function.

branch points $\pm N, \pm N|\cos \theta|$ are taken as extending vertically upwards, as shown in figure 6 . Thus, the phase of complex square roots of the form $\left(\omega^{2}-\omega_{0}^{2}\right)^{\frac{1}{2}}$ has the following behaviour on the real axis:

$$
\begin{aligned}
\operatorname{ph}\left(\omega^{2}-\omega_{0}^{2}\right)^{\frac{1}{2}} & =0 & & \left(\omega>\omega_{0}\right), \\
& =-\frac{1}{2} \pi & & \left(|\omega|<\omega_{0}\right), \\
& =-\pi & & \left(\omega<-\omega_{0}\right) .
\end{aligned}
$$

Denoting from now on complex square roots, defined by (5.3), as powers $\frac{1}{2}$ in curly 
brackets and real square roots as powers $\frac{1}{2}$ in square brackets, we obtain for the Green's function at every real frequency

$$
\begin{aligned}
G(\boldsymbol{r}, \omega) & =\frac{1}{4 \pi r} \frac{\exp \left(-\frac{\beta r}{2}\left[\frac{\omega^{2}-N^{2} \cos ^{2} \theta}{\omega^{2}-N^{2}}\right]^{\frac{1}{2}}\right)}{\left[\left(\omega^{2}-N^{2}\right)\left(\omega^{2}-N^{2} \cos ^{2} \theta\right)\right]^{\frac{1}{2}}} \quad(|\omega|>N) \\
& =\mathrm{i} \frac{\operatorname{sgn} \omega}{4 \pi r} \frac{\exp \left(-\mathrm{i} \frac{\beta r}{2}\left[\frac{\omega^{2}-N^{2} \cos ^{2} \theta}{N^{2}-\omega^{2}}\right]^{\frac{1}{2}} \operatorname{sgn} \omega\right)}{\left[\left(N^{2}-\omega^{2}\right)\left(\omega^{2}-N^{2} \cos ^{2} \theta\right)\right]^{\frac{1}{2}}} \quad(N|\cos \theta|<|\omega|<N) \\
& =-\frac{1}{4 \pi r} \frac{\exp \left(-\frac{\beta r}{2}\left[\frac{N^{2} \cos ^{2} \theta-\omega^{2}}{N^{2}-\omega^{2}}\right]^{\frac{1}{2}}\right)}{\left[\left(N^{2}-\omega^{2}\right)\left(N^{2} \cos ^{2} \theta-\omega^{2}\right)\right]^{\frac{1}{2}}} \quad(|\omega|<N|\cos \theta|) .
\end{aligned}
$$

In accordance with the group velocity theory, internal waves propagate for frequencies $N|\cos \theta|<|\omega|<N$ and possess there the phase (4.13). Outside this frequency band they are evanescent. Under the Boussinesq approximation their wavy character disappears, apart from a phase jump of $\frac{1}{2} \pi$ on the characteristic cone $|\cos \theta|=|\omega| / N$, where the Green's function diverges. Although this phenomenon is consistent with the confinement of Boussinesq internal waves on the characteristic cone, it also shows the inadequacy of the Green's function to represent them there.

The preceding method was introduced by Pierce (1963), later rederived by Hurley (1972) and recently developed by Appleby \& Crighton (1986, 1987). 'Classical' investigations of the monochromatic Green's function of internal waves involve, however, a somewhat different Fourier analysis in the space domain. $G(\boldsymbol{k}, \omega)$, defined by

$$
G(\boldsymbol{r}, t)=\frac{1}{(2 \pi)^{4}} \nsucc \mathrm{d} \omega \int \mathrm{d}^{3} k G(\boldsymbol{k}, \omega) \mathrm{e}^{\mathrm{i}(\omega t-\boldsymbol{k} \cdot \boldsymbol{r})},
$$

readily follows from $(3.15)$ as

$$
G(\boldsymbol{k}, \omega)=\frac{1}{\omega^{2}\left(k^{2}+\frac{1}{4} \beta^{2}\right)-\bar{N}^{2} k_{\mathrm{h}}^{2}},
$$

but remains indeterminate for frequencies and wavenumbers related by the dispersion relation (4.9). The radiation condition makes it determinate, by imposing that

$$
\begin{aligned}
G(\boldsymbol{k}, \omega) & =\lim _{\epsilon \rightarrow 0_{+}} \frac{1}{(\omega-\mathrm{i} \epsilon)^{2}\left(k^{2}+\frac{1}{4} \beta^{2}\right)-N^{2} k_{\mathrm{h}}^{2}}, \\
& =\operatorname{pv} \frac{1}{\omega^{2}\left(k^{2}+\frac{1}{4} \beta^{2}\right)-N^{2} k_{\mathrm{h}}^{2}}+\mathrm{i} \pi \delta\left[\omega^{2}\left(k^{2}+\frac{1}{4} \beta^{2}\right)-N^{2} k_{\mathrm{h}}^{2}\right] \operatorname{sgn} \omega .
\end{aligned}
$$

The principal value of the singular generalized function (5.6) is consequently added to a combination of free waves represented by the Dirac delta, so as to eliminate any wave which does not originate at the source.

$G(k, \omega)$ is first inverted along one space direction by the joint use of the residue theorem and the radiation condition. The passage to (5.4) reduces then to the evaluation of Sonine-Gegenbauer integrals, most of which are given by Watson (1966, §13.47), while others have been especially calculated for the purpose of this 
study. Further detail about this procedure may be found in Voisin (1991). We just deal here with some intermediate results, i.e. $G\left(\boldsymbol{r}_{\mathrm{h}}, k_{z}, \omega\right), G\left(\boldsymbol{k}_{\mathrm{h}}, z, \omega\right)$ and $G\left(k_{x}, y, z, \omega\right)$, of special interest for some problems of internal wave generation, such as moving point sources.

Pierce's (1963) procedure enables their rapid derivation, in the following way: changes of coordinates analogous to (5.1) reduce the equations which define them to one- or two-dimensional Helmholtz-like equations, whose Green's functions have been given by Bleistein (1984, p. 177). In the original coordinate system we obtain

$$
\begin{gathered}
G\left(r_{\mathrm{h}}, k_{z}, \omega\right)=\frac{1}{2 \pi\left(\omega^{2}-N^{2}\right)} K_{0}\left[\frac{\omega}{\left\{\omega^{2}-N^{2}\right\}^{\frac{1}{2}}}\left(k_{z}^{2}+\frac{1}{4} \beta^{2}\right)^{\frac{1}{2}} r_{\mathrm{h}}\right], \\
G\left(k_{\mathrm{h}}, z, \omega\right)=\frac{\exp \left[-\left\{\frac{\omega^{2}-N^{2}}{\omega^{2}} k_{\mathrm{h}}^{2}+\frac{1}{4} \beta^{2}\right\}^{\frac{1}{2}}|z|\right]}{2 \omega\left\{\left(\omega^{2}-N^{2}\right) k_{\mathrm{h}}^{2}+\frac{1}{4} \omega^{2} \beta^{2}\right\}^{\frac{1}{2}}}, \\
G\left(k_{x}, y, z, \omega\right)=\frac{1}{2 \pi \omega\left\{\omega^{2}-N^{2}\right\}^{\frac{1}{2}}} K_{0}\left[\left\{k_{x}^{2}+\frac{1}{4} \beta^{2} \frac{\omega^{2}}{\omega^{2}-N^{2}}\right\}^{\frac{1}{2}}\left\{y^{2}+\frac{\omega^{2}-N^{2}}{\omega^{2}} z^{2}\right\}^{\frac{1}{2}}\right] .
\end{gathered}
$$

Most calculations of the monochromatic Green's function found in the literature are based upon the 'classical' method, in both Boussinesq and non-Boussinesq cases. Ramachandra Rao (1973, 1975), Grigor'ev \& Dokuchaev (1970) and Gorodtsov \& Teodorovich (1983) derived $G\left(\boldsymbol{r}_{\mathrm{h}}, k_{z}, \omega\right)$ in this way. $G\left(\boldsymbol{k}_{\mathrm{h}}, z, \omega\right)$ has been considered by Sarma \& Naidu (1972a,b), Ramachandra Rao (1973), Tolstoy (1973, §7.3), Rehm \& Radt (1975), and also Miles (1971) and Sturova (1980). Gorodtsov \& Teodorovich (1980) eventually dealt with $G\left(k_{x}, y, z, \omega\right)$. In fact, these investigations are often devoted to the pressure and displacement fields generated by point mass and force sources rather than the Green's function defined in (3.15). These fields readily follow from the Green's function, the corresponding calculations being performed in Appendix A. The systematic comparison undertaken there shows published results to coincide with ours, except those of Sarma \& Naidu $(1972 a, b)$, Tolstoy $(1973, \$ 7.3)$ and Ramachandra Rao (1975).

The explanation for these discrepancies lies entirely in the radiation condition. Sarma \& Naidu $(1972 a, b)$ do not remove the exponential density factor (3.9) from the pressure before applying Fourier analysis. Thus, they consider the Fourier transform of a function which does not possess any such transform, even as a generalized function. No radiation condition is needed, but the pressure they obtain is physically unacceptable. Ramachandra Rao (1975) restores the extraction of the density factor, but his application of Sommerfeld's radiation condition seems erroneous and accordingly the pressure he derives for a force source is not consistent with his previous result for a mass source (Ramachandra Rao 1973). Similarly, the difference between Tolstoy's $(1973, \S 7.3)$ displacement and ours is caused by an invalid use of Lighthill's radiation condition, which Tolstoy understands as an attenuation of the radiated waves with time.

\subsection{Impulsive Green's function}

To calculate the impulsive Green's function Sekerzh-Zen'kovich $(1979,1981)$ introduced a direct procedure, based upon

$$
G(\boldsymbol{k}, t)=-\frac{H(t)}{N k_{\mathrm{h}}\left(k^{2}+\frac{1}{4} \beta^{2}\right)^{\frac{1}{2}}} \sin \left[N t \frac{k_{\mathrm{h}}}{\left(k^{2}+\frac{1}{4} \beta^{2}\right)^{\frac{1}{2}}}\right],
$$


which follows from the application of residue theorem to $(5.6) . H(t)$ denotes the Heaviside step $(H(t)=1$ for $t>0,0$ for $t<0)$. When the Boussinesq approximation is made, Fourier inverting (5.11) in space yields an integral expression of the Green's function, rewritten by Teodorovich \& Gorodtsov (1980) as the spectral decomposition

$$
G_{\mathrm{B}}(\boldsymbol{r}, t)=-\frac{H(t)}{2 \pi^{2} r} \int_{N|\cos \theta|}^{N} \frac{\sin \omega t}{\left[\left(N^{2}-\omega^{2}\right)\left(\omega^{2}-N^{2} \cos ^{2} \theta\right)\right]^{\frac{1}{2}}} \mathrm{~d} \omega .
$$

Hereafter a subscript B will denote a Boussinesq result. Thus, impulsive internal waves can be expressed as the superposition only of propagating monochromatic waves.

Clearly, a procedure relating more explicitly the monochromatic Green's function to the impulsive one, and holding for non-Boussinesq as well as Boussinesq internal waves, is needed. It begins by Fourier inverting (5.2) in time, so that (the integration contour is sketched in figure 6 and lies below the singularities of the integrand)

$$
G(\boldsymbol{r}, t)=\frac{1}{8 \pi^{2} r} \Psi_{-\infty}^{+\infty} \frac{\exp \left[\mathrm{i} \omega t-\frac{\beta r}{2}\left\{\frac{\omega^{2}-N^{2} \cos ^{2} \theta}{\omega^{2}-N^{2}}\right\}^{\frac{1}{2}}\right]}{\left\{\omega^{2}-N^{2}\right\}^{\frac{1}{2}}\left\{\omega^{2}-N^{2} \cos ^{2} \theta\right\}^{\frac{1}{2}}} \mathrm{~d} \omega .
$$

Then, separating by (5.4) the contributions of propagating and evanescent waves, we find

$$
\begin{aligned}
G(\boldsymbol{r}, t)=\frac{1}{4 \pi^{2} r} \int_{N|\cos \theta|}^{N} & \frac{\sin \left(\frac{\beta r}{2}\left[\frac{\omega^{2}-N^{2} \cos ^{2} \theta}{N^{2}-\omega^{2}}\right]^{\frac{1}{2}}-\omega t\right)}{\left[\left(N^{2}-\omega^{2}\right)\left(\omega^{2}-N^{2} \cos ^{2} \theta\right)\right]^{\frac{1}{2}}} \mathrm{~d} \omega \\
& +\frac{1}{4 \pi^{2} r}\left(\int_{N}^{\infty}-\int_{0}^{N|\cos \theta|}\right) \frac{\exp \left(-\frac{\beta r}{2}\left[\frac{\omega^{2}-N^{2} \cos ^{2} \theta}{\omega^{2}-N^{2}}\right]^{\frac{1}{2}}\right) \cos \omega t}{\left[\left(\omega^{2}-N^{2}\right)\left(\omega^{2}-N^{2} \cos ^{2} \theta\right)\right]^{\frac{1}{2}}} \mathrm{~d} \omega .
\end{aligned}
$$

Closing for $t<0$ the integration contour of (5.13) by an infinite semicircle in the lower half-plane where $G(r, \omega)$ is analytic, and applying Jordan's lemma, we recover the causality of the Green's function: $G(\boldsymbol{r}, t)=0$ for $t<0$. Separating its odd and even parts with respect to time, we have

$$
G(r, t)=2 H(t) G_{\mathrm{odd}}(r, t)=2 H(t) G_{\mathrm{even}}(r, t),
$$

and (5.14) accordingly becomes

$$
G(r, t)=-\frac{H(t)}{2 \pi^{2} r} \int_{N|\cos \theta|}^{N} \frac{\cos \left(\frac{\beta r}{2}\left[\frac{\omega^{2}-N^{2} \cos ^{2} \theta}{N^{2}-\omega^{2}}\right]^{\frac{1}{2}}\right) \sin \omega t}{\left[\left(N^{2}-\omega^{2}\right)\left(\omega^{2}-N^{2} \cos ^{2} \theta\right)\right]^{\frac{1}{2}}} \mathrm{~d} \omega .
$$

Evanescent waves consequently merge with propagating waves and transform them into the standing waves appearing in (5.16). Under the Boussinesq approximation, the relative contributions of propagating and evanescent waves are even equal, but it must be remembered that their propagating or evanescent character is lost in that case.

Closing now for $t>0$ the integration contour of (5.13) in the upper half-plane transforms the Green's function into the sum of four integrals along the branch cuts emanating from $\pm N, \pm N|\cos \theta|$ (cf. figure 6). Impulsive internal waves are thus made up of the combination of gravity waves, that is plane propagating waves of frequency 
$N|\cos \theta|$, and buoyancy oscillations, which are oscillations of the fluid at frequency $N$ (these names date back to Dickinson 1969). In $\$ 4.1$ group velocity allowed a first examination of the properties of both components. Unfortunately neither these properties, nor the way that gravity waves and buoyancy oscillations are combined to form the internal wave field, are exhibited by the spectral integral (5.16). Thus alternative procedures, of either exact or asymptotic nature, must be developed for the calculation of the impulsive Green's function. This is performed in $\$ 6$.

\section{Asymptotic Green's function}

\subsection{Boussinesq Green's function}

Under the Boussinesq approximation, the monochromatic Green's function

$$
G_{\mathrm{B}}(r, \omega)=\frac{1}{4 \pi r} \frac{1}{\left\{\omega^{2}-N^{2}\right\}^{\frac{1}{2}}\left\{\omega^{2}-N^{2} \cos ^{2} \theta\right\}^{\frac{1}{2}}}
$$

is made up of the product of two functions whose inverse Fourier transforms are Bessel functions (see (D 6) in Appendix D). Then convolution theorem implies that

$$
G_{\mathrm{B}}(r, t)=-\frac{H(t)}{4 \pi r} \int_{0}^{t} J_{0}(N \tau|\cos \theta|) J_{0}[N(t-\tau)] \mathrm{d} \tau,
$$

a time decomposition of the impulsive Green's function showing how gravity waves and buoyancy oscillations interact to produce the overall internal wave pattern. At the vertical from the source and at its level only the latter ones are encountered, according to

$$
\begin{aligned}
& \left.G_{\mathrm{B}}\right|_{r_{\mathrm{h}}=0}=-H(t) \frac{\sin N t}{4 \pi N|z|}, \\
& \left.\frac{\partial G_{\mathrm{B}}}{\partial t}\right|_{z=0}=-H(t) \frac{J_{0}(N t)}{4 \pi r_{\mathrm{h}}},
\end{aligned}
$$

which follow from the application of (D 5) and (D 6) (Appendix D) to (6.1). Instead of being confined at the vertical from the source, buoyancy oscillations are consequently distributed over the whole of space, in contradiction with the predictions of the group velocity theory.

To gain a better physical insight into the separation of internal waves into gravity waves and buoyancy oscillations, we must resort to asymptotic procedures, deduced from general theorems about Fourier transforms. The small-time expansion of $G_{\mathrm{B}}(\boldsymbol{r}, t)$ is obtained by term-by-term inversion of the high-frequency expansion of $G_{B}(r, \omega)$ (Morse \& Feshbach 1953, p. 462). Similarly a large-time expansion of $G_{\mathrm{B}}(r, t)$ is derived by adding up the contributions of the singularities $\pm N, \pm N|\cos \theta|$ of $G_{\mathrm{B}}(r, \omega)$ (Lighthill 1958, §4.3). Such an analysis was first applied to the Green's function of internal waves by Dickinson (1969), in the context of Laplace transforms.

For small times $N t \ll 1$, expanding $G_{\mathbf{B}}(r, \omega)$ in an inverse power series of $\omega$ and allowing for (D 2), we obtain

where

$$
\begin{gathered}
G_{\mathrm{B}}(r, t) \sim-\frac{H(t)}{4 \pi N r} \sum_{n=0}^{\infty} \alpha_{n} \frac{(N t)^{2 n+1}}{(2 n+1) !} \\
\alpha_{n}=\frac{(-1)^{n}}{\pi} \sum_{j+k=n} \frac{\Gamma\left(j+\frac{1}{2}\right) \Gamma\left(k+\frac{1}{2}\right)}{j ! k !} \cos ^{2 k} \theta
\end{gathered}
$$


The series (6.5) converges for all finite $t$, so constituting an exact representation of the impulsive Green's function rather than a mere expansion. To leading order,

$$
G_{\mathrm{B}}(r, t) \sim-H(t) \frac{t}{4 \pi r} .
$$

In agreement with Batchelor's $(1967, \S 6.10)$ general discussion, and Morgan's (1953) discussion of the analogous case of unstratified rotating fluids, the Boussinesq fluid initially ignores its stratification. Thus its motion, described by (6.7), is irrotational. Further details about the question of initial conditions for stratified or rotating fluids, which has been a controversial subject for several years, are given in Appendix B.

For large times $N t \gg 1$, gravity waves and buoyancy oscillations have become separated. They respectively correspond to the contributions of the pairs of branch points $\pm N|\cos \theta|$ and $\pm N$. Gravity waves are, from the expansion of $G_{\mathrm{B}}(r, \omega)$ in powers of $\{\omega-N|\cos \theta|\}^{\frac{1}{2}}$ near $N|\cos \theta|$ and its Fourier inversion by (D 3),

with

$$
G_{\mathrm{B}_{\mathrm{g}}}(r, t) \sim-\frac{H(t)}{(2 \pi)^{\frac{3}{2}} N r \sin \theta} \operatorname{Re}\left[\frac{\mathrm{e}^{\mathrm{i}(N t|\cos \theta|-\pi / 4)}}{(N t|\cos \theta|)^{\frac{1}{2}}} \sum_{n=0}^{\infty} \mathrm{i}^{n} \frac{\Gamma\left(n+\frac{1}{2}\right)}{\pi^{\frac{1}{2}}} \frac{\beta_{n}}{(N t|\cos \theta|)^{n}}\right],
$$

$$
\beta_{n}=\frac{(-1)^{n}}{\pi^{\frac{3}{2}}} \sum_{j+k+m=n} \frac{\Gamma\left(j+\frac{1}{2}\right) \Gamma\left(k+\frac{1}{2}\right) \Gamma\left(m+\frac{1}{2}\right)}{j ! k ! m !} \frac{(-1)^{m}|\cos \theta|^{k+m}}{2^{j}(1+|\cos \theta|)^{k}(1-|\cos \theta|)^{m}} .
$$

The conjugate contribution of $-N|\cos \theta|$ has been incorporated by taking two times the real part of the result. We similarly have for buoyancy oscillations

with

$$
G_{\mathrm{B}_{\mathrm{b}}}(\boldsymbol{r}, t) \sim-\frac{H(t)}{(2 \pi)^{\frac{3}{2}} N r \sin \theta} \operatorname{Im}\left[\frac{\mathrm{e}^{\mathrm{i}(N t-\pi / 4)}}{(N t)^{\frac{1}{2}}} \sum_{n=0}^{\infty} \mathrm{i}^{n} \frac{\Gamma\left(n+\frac{1}{2}\right)}{\pi^{\frac{1}{2}}} \frac{\gamma_{n}}{(N t)^{n}}\right]
$$

$$
\gamma_{n}=\frac{(-1)^{n}}{\pi^{\frac{3}{2}}} \sum_{j+k+m=n} \frac{\Gamma\left(j+\frac{1}{2}\right) \Gamma\left(k+\frac{1}{2}\right) \Gamma\left(m+\frac{1}{2}\right)}{j ! k ! m !} \frac{1}{2^{j}(1+|\cos \theta|)^{k}(1-|\cos \theta|)^{m}} .
$$

To better exhibit the angular dependence of the coefficients $\beta_{n}$ and $\gamma_{n}$ it may be preferred to express them in terms of generating functions, in which case

$$
\left(\beta_{n}, \gamma_{n}\right)=\sum_{j+k=n} \frac{(-1)^{j}}{j !} \frac{\Gamma\left(j+\frac{1}{2}\right)}{\pi^{\frac{1}{2}}} \frac{\left(f_{k}, g_{k}\right)}{2^{j}}
$$

where

$$
\begin{aligned}
& \left(1-2 \frac{\mu}{\tan ^{2} \theta}-\frac{\mu^{2}}{\tan ^{2} \theta}\right)^{-\frac{1}{2}}=\sum_{k=0}^{\infty} f_{k} \mu^{k}, \\
& \left(1+2 \frac{\mu}{\sin ^{2} \theta}+\frac{\mu^{2}}{\sin ^{2} \theta}\right)^{-\frac{1}{2}}=\sum_{k=0}^{\infty} g_{k} \mu^{k}
\end{aligned}
$$

Of crucial importance is the leading-order term of the large-time expansion,

$$
G_{\mathrm{B}}(r, t) \sim-\frac{H(t)}{(2 \pi)^{\frac{3}{2}} N r \sin \theta}\left[\frac{\cos \left(N t|\cos \theta|-\frac{1}{4} \pi\right)}{(N t|\cos \theta|)^{\frac{1}{2}}}+\frac{\sin \left(N t-\frac{1}{4} \pi\right)}{(N t)^{\frac{1}{2}}}\right],
$$

which extends Lighthill's (1978) equations (255)-(258) to the Green's function of internal waves, by simultaneously including gravity waves and buoyancy oscillations. Gravity waves are verified to have the phase expected from the consideration of group velocity. Buoyancy oscillations are confirmed not to propagate, but also to be present everywhere. For the moment we just note these 
points, and postpone the full interpretation of (6.15) until \$7.2. Another asymptotic expansion of the Green's function, alternative to (6.15), may also be derived; this is the matter of Appendix C.

When the vertical line passing through the source $(\theta=0$ or $\pi)$ or the horizontal plane containing it $\left(\theta=\frac{1}{2} \pi\right)$ are approached, (6.15) is invalidated. Near to the vertical, gravity waves and buoyancy oscillations may no longer be separated and combine to form the persistent oscillation accounted for by (6.3). Near to the horizontal, (6.4) shows gravity waves, whose frequency tends towards zero, to vanish. Only buoyancy oscillations remain, still described by the corresponding terms of (6.15). A more precise study of the range of validity of large-time expansions of internal waves has been achieved by Zavol'skii \& Zaitsev (1984). Its consideration is, again, postponed until $\$ 7.2$.

\subsection{Non-Boussinesq Green's function}

Non-Boussinesq effects induce in the phase of the impulsive Green's function (5.13) an additional dependence upon position $r$ and frequency $\omega$ which significantly alters the way that its asymptotic expansions are obtained. First, the calculation of a general form for all terms is excluded and we shall only consider leading-order terms.

For small times $N t \ll 1$, the procedure of $\$ 6.1$ remains applicable and yields

$$
G(r, t) \sim-H(t) \frac{\mathrm{e}^{-\beta r / 2}}{4 \pi r} t .
$$

Surprisingly, non-Boussinesq effects cause an isotropic exponential decrease of the perturbation with distance from the source, to be added to the anisotropic exponential variation (3.9). When $r$ is small compared with the scale height $2 / \beta$ of the stratification the Boussinesq result (6.7) is recovered and the initial motion is irrotational again.

For large times $N t \gg 1$, two distinct types of expansions must be separated, depending on whether $r$ is allowed to become large with $t$ or not: (i) $t \rightarrow \infty$ with $r / t$ fixed, (ii) $t \rightarrow \infty$ with $r$ fixed. They correspond to different regions of space and time which, as Bretherton (1967) pointed out in a similar case, 'merge into one another, in those places where the respective asymptotic expansions have common areas of validity'. Expansion (i) describes a given wavepacket moving at the group velocity $c_{\mathrm{g}}=r / t$, so as to maintain its frequency and wavenumber constant. At a given point expansion (i) is first valid, and the successive arrivals of wavepackets of decreasing group velocity are observed; then, as $c_{\mathrm{g}} \rightarrow 0$ and $t / r \rightarrow \infty$, expansion (ii) becomes relevant. Conversely at a given (but still large) time, region (ii) is composed of points situated at finite distances from the source, while region (i) lies outside it.

For $N t \gg 1$ and a fixed $r / t$, the impulsive Green's function (5.13) may be evaluated by a graphical procedure, developed by Felsen (1969) and applied by Liu \& Yeh (1971) to acoustic-gravity waves. This procedure is based upon the method of stationary phase (e.g. Bleistein $1984, \S 2.7$ ). The phase

$$
\Phi(\omega)=\omega t+\mathrm{i} \frac{\beta r}{2}\left\{\frac{\omega^{2}-N^{2} \cos ^{2} \theta}{\omega^{2}-N^{2}}\right\}^{\frac{1}{2}} \equiv \omega t-\xi(\omega) r
$$

of the integrand is stationary for the frequency $\omega_{\mathrm{s}}$, with which is associated a wavepacket of radial wavenumber $\xi\left(\omega_{\mathrm{s}}\right)$ and group velocity $c_{\mathbf{g}}\left(\omega_{\mathrm{s}}\right)$, verifying

$$
\Phi^{\prime}\left(\omega_{\mathrm{s}}\right)=0 \quad \text { or } \quad \frac{t}{r}=\xi^{\prime}\left(\omega_{\mathrm{s}}\right)=\frac{1}{c_{\mathrm{g}}\left(\omega_{\mathrm{s}}\right)} .
$$




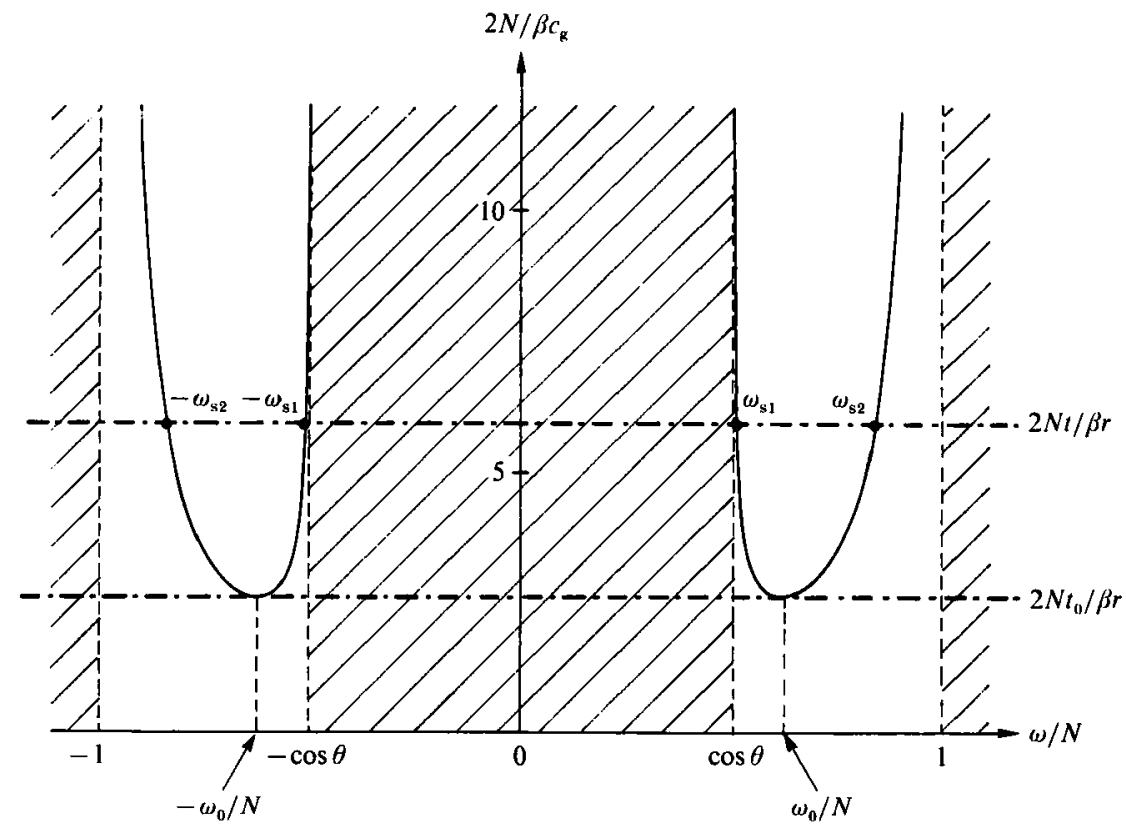

FigURE 7. Graphical determination of the frequencies $\omega_{\mathrm{s} 1}$ of gravity waves and $\omega_{\mathrm{s} 2}$ of buoyancy oscillations, for propagation from a point source along a direction inclined at $\theta=60^{\circ}$ to the vertical.

The dominant contributions to the integral are assumed to arise from the frequency band $M|\cos \theta|<|\omega|<N$ of propagating internal waves, the group velocity of which (4.14) has already been plotted in figure 4 versus frequency. Intersecting a similar plot of the inverse group velocity in figure 7 with horizontal lines of heights $t / r$ provides, according to (6.18), a qualitative description of the internal waves received at a fixed point versus time.

Thus, at time

$$
t_{0}=\frac{\beta r}{2} \frac{N^{2} \omega_{0} \sin ^{2} \theta}{\left[N^{2}-\omega_{0}^{2}\right]^{\frac{3}{2}}\left[\omega_{0}^{2}-N^{2} \cos ^{2} \theta\right]^{\frac{1}{2}}},
$$

the first internal waves of frequency $\omega_{0}$ given by (4.15) and maximum group velocity $c_{\mathrm{g} 0}=r / t_{0}$ reach the point under consideration. The structure of the associated wavefront was investigated by Kato $(1966 b)$ and Cole \& Greifinger (1969) for acoustic-gravity waves, Mowbray \& Rarity (1967) and Tolstoy $(1973, \$ 7.3)$ for nonBoussinesq internal waves. It is in fact a caustic: internal waves gradually build up just prior to its arrival, and subsequently separate into two components of frequencies $\omega_{\mathrm{s} 1}$ and $\omega_{\mathrm{s} 2}$ such that $M|\cos \theta|<\omega_{\mathrm{s} 1}<\omega_{0}$ and $\omega_{0}<\omega_{\mathrm{s} 2}<N$. When $t / r \rightarrow \infty$, $\omega_{\mathrm{s} 1}$ and $\omega_{\mathrm{s} 2}$ respectively tend towards $N|\cos \theta|$ and $N$, identifying the corresponding components as gravity waves and buoyancy oscillations. Ultimately, the Boussinesq situation is recovered.

Not only this qualitative insight into the propagation of internal waves, but also the related quantitative analysis, are provided by Felsen's (1969) method. Accordingly, the build-up of internal waves prior to the arrival of the caustic, and their subsequent decomposition into gravity waves and buoyancy oscillations, are accounted for by an Airy function; the following evolution of both components is described by the standard stationary-phase formula. Unfortunately, these results rely on the graphical determination of the stationary frequencies $\omega_{\mathrm{s} 1}$ and $\omega_{\mathrm{s} 2}$ and 
cannot be written in explicit form, except in the final stage $t / r \rightarrow \infty$, when analytical expressions are available for $\omega_{\mathrm{s} 1}$ and $\omega_{\mathrm{s} 2}$.

We now focus attention on that stage, when region (ii) is entered : $N t \gg 1$ and $\beta r$ is held fixed. To avoid the neglect of evanescent internal waves, we make no further use of Felsen's approach and rather remark that, in region (ii), expanding the impulsive Green's function is again expanding an inverse Fourier transform for large times. Thus, Lighthill's (1958) asymptotic method is relevant again. Gravity waves and buoyancy oscillations arise as, respectively, the contributions of the pairs of branch points $\pm N|\cos \theta|$ and $\pm N$ of the monochromatic Green's function (5.2). Gravity waves are, from the expansion of $G(r, \omega)$ in terms of $\{\omega-N|\cos \theta|\}^{\frac{1}{2}}$ and its inversion by (D 8),

$$
G_{\mathrm{g}}(\boldsymbol{r}, t) \sim-\frac{H(t)}{(2 \pi)^{\frac{3}{2}} N r \sin \theta} \frac{\cos \left[\left(N t-\frac{\beta^{2} r^{2}}{8 N t \sin ^{2} \theta}\right)|\cos \theta|-\frac{1}{4} \pi\right]}{(N t|\cos \theta|)^{\frac{1}{2}}} .
$$

Similarly buoyancy oscillations are reducible to the inverse transform (D 9), of which (D 15) provides an asymptotic expansion, so that

$$
\begin{aligned}
G_{\mathrm{b}}(\boldsymbol{r}, t) \sim- & \frac{H(t)}{(2 \pi)^{\frac{3}{2}} \sqrt{ } 3 N \sin \theta}\left\{\frac{\sin \left[N t-\frac{3}{2}\left(\frac{1}{2} \beta r \sin \theta\right)^{\frac{2}{3}}(N t)^{\frac{1}{3}}-\frac{1}{4} \pi\right]}{(N t)^{\frac{1}{2}}}\right. \\
& \left.+\exp \left[-\frac{3 \sqrt{ } 3}{4}\left(\frac{1}{2} \beta r \sin \theta\right)^{\frac{2}{3}}(N t)^{\frac{1}{3}}\right] \frac{\sin \left[N t+\frac{3}{4}\left(\frac{1}{2} \beta r \sin \theta\right)^{\frac{2}{3}}(N t)^{\frac{1}{3}}-\frac{1}{4} \pi\right]}{(N t)^{\frac{1}{2}}}\right\} .
\end{aligned}
$$

As in the Boussinesq case an alternative expression of gravity waves may be proposed; its derivation is outlined in Appendix C.

Buoyancy oscillations are now waves, to which non-Boussinesq effects have given some propagation. Contrary to gravity waves, they comprise both propagating and evanescent internal waves. However, as long as the Boussinesq approximation is not made, evanescent waves decay exponentially with time and stay negligible. In $\$ 7.3$ we shall carry the interpretation of gravity and buoyancy waves further, by calculating their characteristics (frequencies and wavevectors).

Ultimately both waves become Boussinesq, as

$$
\frac{1}{2} \beta r \ll N t \sin \theta
$$

for the former, and

$$
\frac{1}{2} \beta r_{\mathrm{h}} \ll N t
$$

for the latter. Instead of the 'classical' near-field argument $\frac{1}{2} \beta|z| \ll 1$ (Hendershott 1969), two surfaces, respectively toroidal and cylindrical, define the validity of the Boussinesq approximation for gravity and buoyancy waves. They expand along the horizontal at velocity $2 N / \beta$ and are represented in figure 8 . Their existence simply reflects the complex structure of time-dependent internal wave fields; the significance of (6.22) and (6.23) remains, in agreement with Lighthill (1978, \$4.2), small wavelengths $\lambda \ll 2 / \beta$.

For buoyancy waves the Boussinesq approximation is moreover non-uniform. As (6.23) becomes valid evanescent waves become comparable with propagating waves until in the end, when non-Boussinesq terms vanish in (6.21), both contributions are the same. Then the buoyancy oscillations appearing in (6.15) are recovered, but multiplied by $2 / \sqrt{ } 3$. Such a non-uniformity is not surprising, since making both $\beta \rightarrow 0$ and $\omega \rightarrow N$ in (5.2) is clearly contradictory. Again we postpone the interpretation of this phenomenon until $\$ 7.3$ and just note, as regards its mathematical significance, that it is due to the coalescence of two saddle points with a branch point of both the 


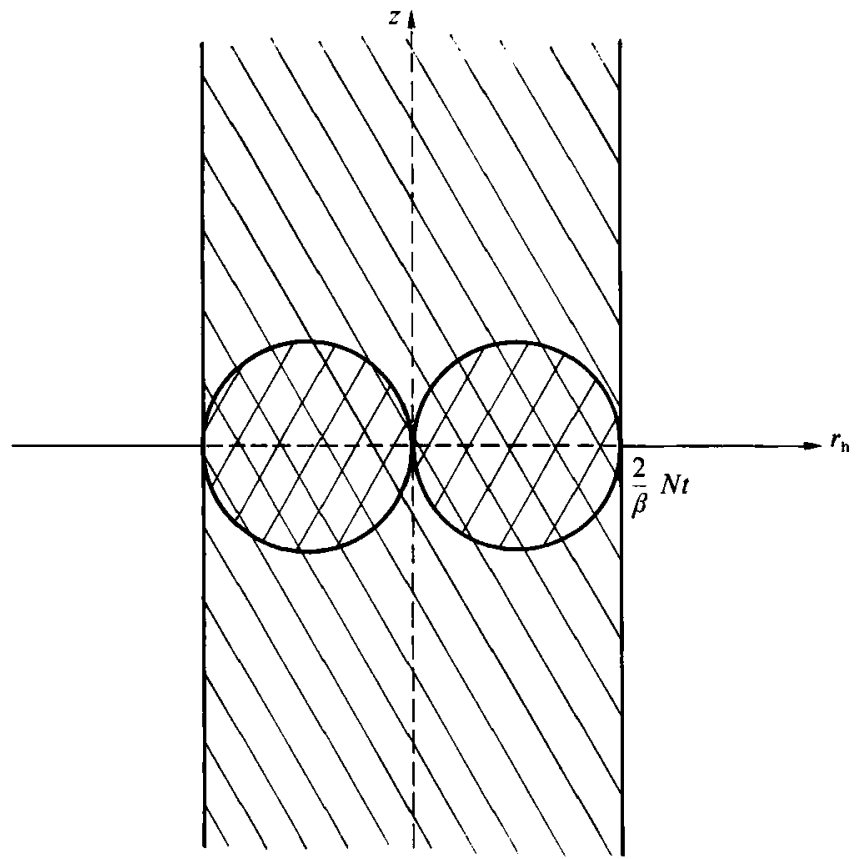

FIGURE 8. Regions for the validity of the Boussinesq approximation, for gravity (a torus of radius $2 N t / \beta$ ) and buoyancy (a cylinder with the same radius) waves. Boussinesq zone for gravity waves; Boussinesq zone for buoyancy oscillations.

argument of the exponent and the amplitude (cf. Appendix D). The uniform asymptotic expansions of Bleistein \& Handelsman (1986, ch. 9) do not seem to apply to this case.

\section{Internal wave field of a point mass source}

As the internal potential generated by a point mass source, the Green's function has no direct physical significance and its interpretation requires the consideration of the associated pressure and velocity fields. In the present section, for an impulsive source $m(\boldsymbol{r}, t)=m_{0} \delta(\boldsymbol{r}) \delta(t)$ releasing a volume $m_{0}$ of fluid, we deduce these fields from the Green's function, by multiplying it by $m_{0}$ and differentiating it according to (3.11)-(3.12). There may, however, be instances where the Green's function has a meaning in itself, such as the Cauchy problem for internal waves (SekerzhZen'kovich 1982); see Appendix B.

\subsection{Monochromatic waves}

Continuing the approach of $\S 5$, we first deal with a monochromatic source, and replace in (3.11) $-(3.12) \partial / \partial t$ by $i \omega$. Then, the differentiation of $G(r, \omega)$ yields

$$
\begin{aligned}
& P(r, \omega)=\mathrm{i} \frac{\rho_{00} m_{0}}{4 \pi r} \omega\left\{\frac{\omega^{2}-N^{2}}{\omega^{2}-N^{2} \cos ^{2} \theta}\right\}^{\frac{1}{2}} \exp \left(-\frac{\beta r}{2}\left\{\frac{\omega^{2}-N^{2} \cos ^{2} \theta}{\omega^{2}-N^{2}}\right\}^{\frac{1}{2}}\right), \\
& v(r, \omega)=\frac{m_{0}}{4 \pi r^{2}} \omega^{2} \frac{\left\{\omega^{2}-N^{2}\right\}^{\frac{1}{2}}}{\left\{\omega^{2}-N^{2} \cos ^{2} \theta\right\}^{\frac{3}{2}}} \exp \left(-\frac{\beta r}{2}\left\{\frac{\omega^{2}-N^{2} \cos ^{2} \theta}{\omega^{2}-N^{2}}\right\}^{\frac{1}{2}}\right) \\
& \times\left\{\left[1+\frac{\beta r}{2}\left\{\frac{\omega^{2}-N^{2} \cos ^{2} \theta}{\omega^{2}-N^{2}}\right\}^{\frac{1}{2}}\right] \frac{r}{r}+\frac{\beta r}{2} \frac{\omega^{2}-N^{2} \cos ^{2} \theta}{\omega^{2}-N^{2}} e_{z}\right\} .
\end{aligned}
$$


Non-Boussinesq effects add a vertical component to the radial motion of the fluid. In the frequency range $M|\cos \theta|<|\omega|<N$ of propagating internal waves the radial and vertical velocities are out of phase, for the term in square brackets in (7.2) is complex. Thus, the trajectories of fluid particles are elliptical, in agreement with the group velocity theory.

Under the Boussinesq approximation the pressure and velocity fields become

$$
\begin{aligned}
& P_{\mathrm{B}}(\boldsymbol{r}, \omega)=\mathrm{i} \frac{\rho_{0} m_{0}}{4 \pi r} \omega\left\{\frac{\omega^{2}-N^{2}}{\omega^{2}-N^{2} \cos ^{2} \theta}\right\}^{\frac{1}{2}}, \\
& \boldsymbol{v}_{\mathrm{B}}(\boldsymbol{r}, \omega)=\frac{m_{0}}{4 \pi r^{2}} \omega^{2} \frac{\left\{\omega^{2}-N^{2}\right\}^{\frac{1}{2}}}{\left\{\omega^{2}-N^{2} \cos ^{2} \theta\right\}^{\frac{3}{2}}} \frac{r}{r} .
\end{aligned}
$$

Consistently with the neglect of density variations $\rho_{00}$ has been replaced by $\rho_{0}$ in (7.3). Fluid particles move as expected along radial trajectories. Out of the characteristic cone the pressure and velocity are $\frac{1}{2} \pi$ out of phase, implying that no energy flux, proportional to $\operatorname{Re}\left[\mathrm{Pv}^{*}\right]$ where ${ }^{*}$ denotes a complex conjugate (see Lighthill 1978, §4.2), is radiated. Energy, and thus internal waves, are confined on the cone, where they diverge. There, point sources are no longer an adequate model of real sources of internal waves. Only the consideration of the finite extent of the latter will account for the radial decrease as $1 / r^{\frac{1}{2}}$, and the transverse phase variations, experimentally exhibited by McLaren et al. (1973).

\subsection{Boussinesq impulsive waves}

Passing to impulsive internal waves we commence as in $\$ 6.1$ by studying the Boussinesq pressure and velocity fields. For small times $N t \ll 1$, either differentiating (6.7) according to (3.11)-(3.12) or asymptotically inverting (7.3)-(7.4) by (D 1), we obtain

$$
\begin{aligned}
& P_{\mathbf{B}}(\boldsymbol{r}, t) \sim \frac{\rho_{0} m_{0}}{4 \pi r} \delta^{\prime}(t), \\
& \boldsymbol{v}_{\mathrm{B}}(\boldsymbol{r}, t) \sim \frac{m_{0}}{4 \pi r^{2}} \frac{r}{r} \delta(t) .
\end{aligned}
$$

The pressure and velocity impulses necessary to set the fluid into motion are recovered (cf. Appendix B).

In similarly differentiating the expansion of the Green's function for large times $N t \gg 1$, care must be taken to retain the first two orders of $(6.8)$ and $(6.10)$ so as to recover the presence of buoyancy oscillations. Lighthill's (1958) method may also be applied to (7.3)-(7.4), in which cases it involves (D 3)-(D 4). Both procedures yield

$$
\begin{gathered}
P_{\mathrm{B}}(r, t) \sim-H(t) \frac{\rho_{0} N^{2} m_{0}}{(2 \pi)^{\frac{3}{2}}}\left[\sin \theta|\cos \theta| \frac{\sin \left(N t|\cos \theta|-\frac{1}{4} \pi\right)}{(N t|\cos \theta|)^{\frac{1}{2}}}-\frac{1}{\sin \theta} \frac{\sin \left(N t-\frac{1}{4} \pi\right)}{(N t)^{\frac{3}{2}}}\right], \\
\boldsymbol{v}_{\mathrm{B}}(\boldsymbol{r}, t) \sim-H(t) \frac{N m_{0}}{(2 \pi)^{\frac{3}{2}} r^{2}} \frac{r}{r}\left[\sin \theta(N t|\cos \theta|)^{\frac{1}{2}} \sin \left(N t|\cos \theta|-\frac{1}{4} \pi\right)+\frac{1}{\sin ^{3} \theta} \frac{\cos \left(N t-\frac{1}{4} \pi\right)}{(N t)^{\frac{3}{2}}}\right] .
\end{gathered}
$$

The phase $\Phi_{\mathrm{g}}=N t|\cos \theta|-\frac{1}{4} \pi$ of gravity waves is, apart from a phase lag of $\frac{1}{4} \pi$, identical to that deduced from the consideration of group velocity. Thus, gravity 
waves propagate as the locally plane waves described in $\$ 4.1$, with the group velocity $c_{\mathrm{g}}=r / t$ and the frequency and wavevector

$$
\begin{gathered}
\omega_{\mathrm{g}}=\frac{\partial \Phi_{\mathrm{g}}}{\partial t}=N|\cos \theta|, \\
\boldsymbol{k}_{\mathrm{g}}=-\nabla \Phi_{\mathrm{g}}=\frac{N t}{r} \frac{\boldsymbol{r}}{r} \times\left(\frac{\boldsymbol{r}}{r} \times \boldsymbol{e}_{z}\right) \operatorname{sgn} z .
\end{gathered}
$$

The pressure and velocity oscillate in phase and verify, consistently with (4.5),

$$
v_{\mathrm{g}} \sim \frac{t}{r} \frac{P_{\mathrm{g}}}{\rho_{\mathrm{0}}} \frac{\boldsymbol{r}}{r}
$$

Note, however, that the velocity grows with time as $t^{\frac{1}{2}}$ and ultimately diverges (Zavol'skii \& Zaitsev 1984), the pressure simultaneously decreasing as $1 / t^{\frac{1}{2}}$ so as to maintain the radiated energy flux finite. Meanwhile, the wavelength $\lambda_{\mathrm{g}}$ given by (4.8) decays as $1 / t$ and may eventually become smaller than intermolecular distances (Sobolev 1965, p. 203), vitiating the continuous medium model. Clearly, a cutoff eliminating the ultimate dominance of the smaller wavelengths is needed (Lighthill 1978, p. 359). As above, the consideration of the finite dimensions of real sources will provide it.

The phase $\Phi_{\mathrm{b}}=N t-\frac{1}{4} \pi$ of buoyancy oscillations confirms that they do not propagate, whereas their amplitude shows them to be present everywhere and to induce a radial motion of fluid particles, in contrast to the predictions of the Boussinesq group velocity theory. This testifies to their non-Boussinesq origin. The pressure and velocity are $\frac{1}{2} \pi$ out of phase, implying a zero energy flux. Compared with gravity waves, buoyancy oscillations decrease with time as $t^{-\frac{3}{2}}$ and remain negligible. This is contradictory to the ultimate dominance of oscillations of the fluid at the buoyancy frequency, experimentally found by McLaren et al. (1973) for transient internal waves. The explanation for this phenomenon lies, again, in the finite size of real sources.

Exact integral expressions of the pressure and velocity fields follow from the same method that has been used to derive the spectral decompositions (5.12) and (5.16) of the Green's function. Amendments are nevertheless required, since (7.4) is not integrable at the singularities $\pm N|\cos \theta|$. A method to circumvent this difficulty, which turns out to be equivalent to that proposed by Zavol'skii \& Zaitsev (1984), may be found in Voisin (1991). It yields for the pressure

$$
P_{\mathrm{B}}(\boldsymbol{r}, t)=\frac{\rho_{0} m_{0}}{2 \pi^{2} r}\left\{\frac{1}{2} \pi \delta^{\prime}(t)+H(t) \int_{N|\cos \theta|}^{N} \omega\left[\frac{N^{2}-\omega^{2}}{\omega^{2}-N^{2} \cos ^{2} \theta}\right]^{\frac{1}{2}} \cos \omega t \mathrm{~d} \omega\right\},
$$

and for the displacement vector $\zeta_{\mathrm{B}}$, related by $v_{\mathrm{B}}=\partial \zeta_{\mathrm{B}} / \partial t$ to the velocity,

$$
\begin{aligned}
\zeta_{\mathrm{B}}(\boldsymbol{r}, t)=H(t) \frac{m_{0}}{2 \pi^{2} r^{2}} \frac{r}{r} & \left\{\frac{1}{2} \pi \cos (N t|\cos \theta|)\right. \\
& \left.+\int_{N|\cos \theta|}^{N} \omega \frac{\left[N^{2}-\omega^{2}\right]^{\frac{1}{2}}}{\left[\omega^{2}-N^{2} \cos ^{2} \theta\right]^{\frac{3}{2}}}[\cos (N t|\cos \theta|)-\cos \omega t] \mathrm{d} \omega\right\} .
\end{aligned}
$$

Zavol'skii \& Zaitsev (1984) compared the numerical calculation of (7.13) with the gravity wave part of its large-time expansion, readily derived from (7.8). They 
concluded that, very early, the asymptotic result is valid, from the level of the source to the conical nodal surface lying closest to the vertical, that is in a region of space which becomes larger and larger as time elapses. After one buoyancy period $(N t / 2 \pi=1)$ for instance, (7.8) induces an error smaller than $5 \%$ for every point verifying $40^{\circ}<\theta<140^{\circ}$. At the same time buoyancy oscillations are confirmed to be negligible in that zone.

\subsection{Non-Boussinesq impulsive waves}

Investigating now the influence of non-Boussinesq effects on impulsive internal waves, we just consider the large-time pressure field. Either differentiating (6.20)-(6.21) according to (3.12) or applying Lighthill's (1958) method to (7.1), in which case (D 8) and (D 15) are involved, we obtain for gravity and buoyancy waves, respectively,

$$
\begin{gathered}
P_{\mathrm{g}}(\boldsymbol{r}, t) \sim-H(t) \frac{\rho_{00} N^{2} m_{0}}{(2 \pi)^{\frac{3}{2}}} \frac{\sin \theta|\cos \theta|}{r} \frac{\sin \left[\left(N t-\frac{\beta^{2} r^{2}}{8 N t \sin ^{2} \theta}\right)|\cos \theta|-\frac{1}{4} \pi\right]}{(N t|\cos \theta|)^{\frac{1}{2}}}, \\
P_{\mathrm{b}}(\boldsymbol{r}, t) \sim H(t) \frac{\rho_{00} N^{2} m_{0}}{(2 \pi)^{\frac{3}{2}} \sqrt{ } 3} \frac{1}{r \sin \theta}\left(\frac{\beta r \sin \theta}{2 N t}\right)^{\frac{2}{3}} \frac{\cos \left[N t-\frac{3}{2}\left(\frac{1}{2} \beta r \sin \theta\right)^{\frac{2}{3}}(N t)^{\frac{1}{3}}-\frac{1}{4} \pi\right]}{(N t)^{\frac{1}{2}}} .
\end{gathered}
$$

In the latter, evanescent waves, which either are negligible compared with propagating waves in the non-Boussinesq case, or equal to them when the Boussinesq situation is reached, have been omitted.

From the phases of gravity and buoyancy waves we deduce their frequencies and wavevectors by differentiation (cf. (7.9)-(7.10)), as

$$
\begin{gathered}
\omega_{\mathrm{g}}=N|\cos \theta|\left[1+\frac{1}{2}\left(\frac{\beta r}{2 N t \sin \theta}\right)^{2}\right], \\
k_{\mathrm{g}_{\mathrm{h}}}=\frac{N t}{r} \sin \theta|\cos \theta| \frac{r_{\mathrm{h}}}{r_{\mathrm{h}}}\left[1-\frac{1}{2}\left(\frac{\beta r}{2 N t \sin \theta}\right)^{2} \frac{3 \cos ^{2} \theta-1}{\sin ^{2} \theta}\right], \\
k_{\mathrm{g}_{z}}=-\frac{N t}{r} \sin ^{2} \theta \operatorname{sgn} z\left[1-\frac{1}{2}\left(\frac{\beta r}{2 N t \sin \theta}\right)^{2} \frac{3 \cos ^{2} \theta+1}{\sin ^{2} \theta}\right], \\
\omega_{\mathrm{b}}=N\left[1-\frac{1}{2}\left(\frac{\beta r_{\mathrm{h}}}{2 N t}\right)^{\frac{2}{3}}\right], \\
k_{\mathrm{b}}=-\frac{N t}{r_{\mathrm{h}}}\left(\frac{\beta r_{\mathrm{h}}}{2 N t}\right)^{\frac{2}{3}} \frac{\boldsymbol{r}_{\mathrm{h}}}{r_{\mathrm{h}}}=-\frac{\beta}{2}\left(\frac{2 N t}{\beta r_{\mathrm{h}}}\right)^{\frac{1}{3}} \frac{r_{\mathrm{h}}}{r_{\mathrm{h}}} .
\end{gathered}
$$

In both cases (4.12) still relates $\omega$ and $k$.

As expected non-Boussinesq effects involve the small parameters $(\beta r) /(2 N t \sin \theta)$ and $\left(\beta r_{\mathrm{h}}\right) /(2 N t)$, and give to buoyancy oscillations a horizontal propagation. To leading order the wavelength of gravity waves reduces to (4.8) and is constant on tori, whereas the wavelength of buoyancy waves,

$$
\lambda_{\mathrm{b}}=\frac{2 \pi}{k_{\mathrm{b}}}=\frac{2 \pi}{N t} r_{\mathrm{h}}\left(\frac{2 N t}{\beta r_{\mathrm{h}}}\right)^{\frac{2}{3}}=\frac{4 \pi}{\beta}\left(\frac{\beta r_{\mathrm{h}}}{2 N t}\right)^{\frac{1}{3}},
$$

is constant on cylinders. Thus, non-Boussinesq transient internal wave fields are 
ruled by two surfaces, a torus and a cylinder, represented in figure 8 . On them the wavelengths of gravity and buoyancy waves are comparable with the scale height $2 / \beta$ of the stratification; well inside them $\lambda \ll 2 / \beta$ and the Boussinesq situation is recovered.

As long as gravity and buoyancy waves remain fully non-Boussinesq, that is near to the cylinder, $\left(\beta r_{\mathrm{h}}\right) /(2 N t)$ is finite and both waves are $O\left((N t)^{-\frac{1}{2}}\right)$. As the cylinder is entered the nature of buoyancy waves changes until eventually, when (6.23) is verified, they become Boussinesq buoyancy oscillations. Then, although their wavelength is zero and their wavenumber infinite (as implied by the second form of $\boldsymbol{k}_{\mathrm{b}}$ ), the spatial variations of their phase are negligible compared with its time variations (as implied by the first form of $\boldsymbol{k}_{\mathrm{b}}$ ). Meanwhile, the leading-order term in their pressure vanishes so that they are now $O\left((N t)^{-\frac{3}{2}}\right)$. Thus, buoyancy waves have simultaneously 'lost' their propagation and become insignificant compared with gravity waves.

No attempt has been made to calculate the non-Boussinesq $O\left((N t)^{-\frac{3}{2}}\right)$ term in (7.15), but it is anticipated that for it the Boussinesq approximation should be uniform and that (7.7) should be recovered as $\left(\beta r_{h}\right) /(2 N t) \rightarrow 0$. This should provide a physical explanation for the non-uniformity of the Boussinesq approximation for buoyancy waves.

\section{Internal wave radiation by a pulsating sphere}

As the simplest example of a source of internal waves with finite dimensions we consider in this section a sphere of radius $a$, on which surface the normal velocity $U(t)$ is imposed. Particular attention will be paid to the far-field modelling of the sphere by the equivalent point mass source, a monopole of strength $4 \pi a^{2} U(t)$ (cf. Pierce 1981, \$\$4.1 and 4.3). From the preceding section, non-Boussinesq effects upon internal wave generation appear to be satisfactorily accounted for by the consideration of point sources. Accordingly, our investigations will be restricted to the Boussinesq case.

Although Hendershott (1969) has already treated both monochromatic and transient Boussinesq pulsations of a sphere, his results are invalidated by the error mentioned in Appendix C. Appleby \& Crighton (1987) also dealt with monochromatic internal waves, and Grimshaw (1969) with transient internal waves, generated by a sphere undergoing monopolar or dipolar motion. The present work is complementary to theirs, in that the link between point and extended sources is investigated.

\subsection{Exact solution}

Under the Boussinesq approximation the internal wave equation (3.10), and the condition of fixed radial velocity at the surface of the sphere, become (subscripts $B$ will from now on be omitted)

$$
\begin{gathered}
\left(\frac{\partial^{2}}{\partial t^{2}} \nabla^{2}+N^{2} \nabla_{\mathrm{h}}^{2}\right) \psi(\boldsymbol{r}, t)=0, \\
\left(\frac{\partial^{2}}{\partial t^{2}} r \frac{\partial}{\partial r}+N^{2} r_{\mathrm{h}} \frac{\partial}{\partial r_{\mathrm{h}}}\right) \psi(\boldsymbol{r}, t)=a U(t) \quad \text { at } \quad r=a .
\end{gathered}
$$

We solve them by the method we used to calculate the Green's function; then we deduce the pressure and velocity fields from the internal potential, by differentiating 


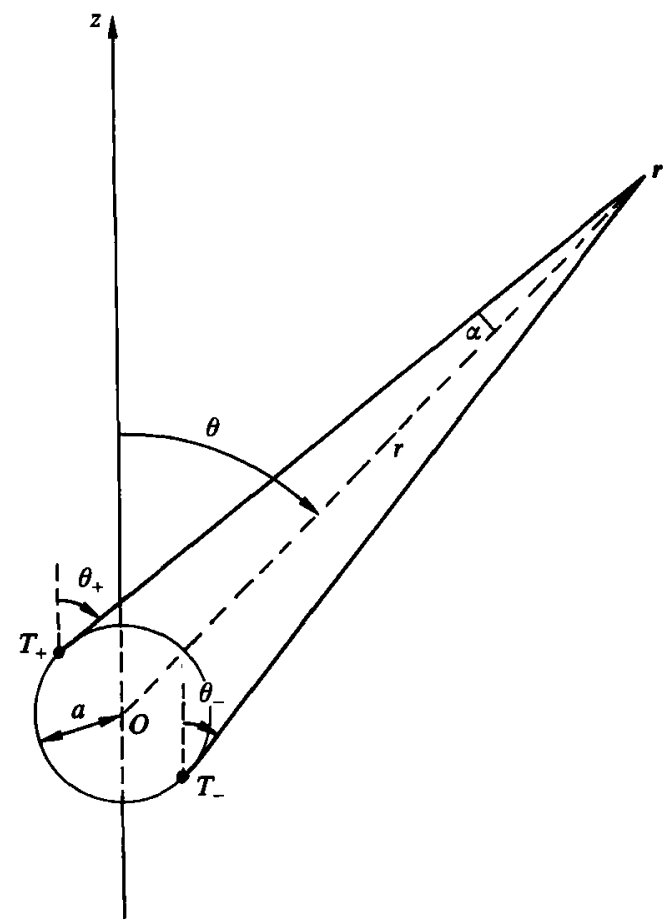

Figure 9. Points of contact $T_{+}$and $T_{-}$of the uppermost and lowermost tangents from a point $r$ to the sphere.

it according to the Boussinesq versions of (3.11)-(3.12). Thus we consider first monochromatic waves, described by

$$
\begin{gathered}
{\left[\left(\omega^{2}-N^{2}\right) \nabla_{\mathrm{h}}^{2}+\omega^{2} \frac{\partial^{2}}{\partial z^{2}}\right] \psi(\boldsymbol{r}, \omega)=0,} \\
{\left[\left(\omega^{2}-N^{2}\right) r_{\mathrm{h}} \frac{\partial}{\partial r_{\mathrm{h}}}+\omega^{2} z \frac{\partial}{\partial z}\right] \psi(r, \omega)=-a U(\omega) \text { at } r=a,}
\end{gathered}
$$

and apply Pierce's (1963) procedure to them.

For $\omega>N$, the change of coordinates (5.1) transforms (8.3) into Laplace's equation and applies (8.4) at the surface of an oblate spheroid, suggesting the introduction of the appropriate spheroidal coordinates (Morse \& Feshbach 1953, p. 662). Stretched oblate spheroidal coordinates, defined by

$$
\begin{gathered}
r_{\mathrm{h}}=a \frac{N}{\omega}\left\{\xi^{2}+1\right\}^{\frac{1}{2}}\left\{1-\eta^{2}\right\}^{\frac{1}{2}}, \\
z=a \frac{N}{\left\{\omega^{2}-N^{2}\right\}^{\frac{1}{2}}} \xi \eta,
\end{gathered}
$$

result from the combination of the two transformations. By introducing the frequencies $\Sigma_{+}$and $\Sigma_{-}$of internal waves emanating from the points of contact $T_{+}$and $T_{-}$of the uppermost and lowermost tangents through the point $r$ to the sphere (figure 9), according to

$$
\Sigma_{ \pm}=N\left|\cos \theta_{ \pm}\right|=N|\cos (\theta \pm \alpha)|=N\left|\left(1-\frac{a^{2}}{r^{2}}\right)^{\frac{1}{2}} \cos \theta \mp \frac{a}{r} \sin \theta\right|
$$


the definition of $\xi$ and $\eta$ is inverted into

$$
\left(\xi^{2}, \eta^{2}\right)= \pm\left\{\left[\frac{\left\{\omega^{2}-\Sigma_{+}^{2}\right\}^{\frac{1}{2}} \pm\left\{\omega^{2}-\Sigma_{-}^{2}\right\}^{\frac{1}{2}}}{2 N}\right]^{2} \frac{r^{2}}{a^{2}}-\cos ^{2} \theta\right\} .
$$

The upper sign is associated to $\xi$ and the lower one to $\eta ; \xi$ is made one-valued by requiring it to be positive as $\omega \rightarrow \infty$. In terms of $\xi$ and $\eta$ we have the differentiation rules

$$
\begin{gathered}
\frac{1}{r_{\mathrm{h}}} \frac{\partial}{\partial r_{\mathrm{h}}}=\frac{1}{r^{2}} \frac{\omega^{2}}{\left\{\omega^{2}-\Sigma_{+}^{2}\right\}^{\frac{1}{2}}\left\{\omega^{2}-\Sigma_{-}^{2}\right\}^{\frac{1}{2}}}\left(\xi \frac{\partial}{\partial \xi}-\eta \frac{\partial}{\partial \eta}\right), \\
\frac{1}{z} \frac{\partial}{\partial z}=\frac{1}{r^{2}} \frac{\omega^{2}-N^{2}}{\left\{\omega^{2}-\Sigma_{+}^{2}\right\}^{\frac{1}{2}}\left\{\omega^{2}-\Sigma_{-}^{2}\right\}^{\frac{1}{2}}}\left(\frac{\xi^{2}+1}{\xi} \frac{\partial}{\partial \xi}+\frac{1-\eta^{2}}{\eta} \frac{\partial}{\partial \eta}\right) .
\end{gathered}
$$

Then the original system of equations (8.3)-(8.4) becomes

$$
\begin{gathered}
\left\{\frac{\partial}{\partial \xi}\left[\left(\xi^{2}+1\right) \frac{\partial}{\partial \xi}\right]+\frac{\partial}{\partial \eta}\left[\left(1-\eta^{2}\right) \frac{\partial}{\partial \eta}\right]\right\} \psi(r, \omega)=0, \\
\frac{\partial}{\partial \xi} \psi(r, \omega)=-\frac{N}{\omega^{2}\left\{\omega^{2}-N^{2}\right\}^{\frac{1}{2}}} a U(\omega) \quad \text { at } \quad \xi=\frac{\left\{\omega^{2}-N^{2}\right\}^{\frac{1}{2}}}{N} .
\end{gathered}
$$

As indicated by (8.10), internal waves do not depend on the 'angular' coordinate $\eta$ because of the monopolar type of motion of the sphere. The internal potential is given by

$$
\psi(\boldsymbol{r}, \omega)=\mathrm{i} \frac{a U(\omega)}{2 N\left\{\omega^{2}-N^{2}\right\}^{\frac{1}{2}}} \ln \frac{\xi-\mathrm{i}}{\xi+\mathrm{i}} .
$$

From its differentiation, and the use of (8.8), the pressure and velocity fields follow as

$$
\begin{gathered}
P(\boldsymbol{r}, \omega)=-\rho_{0} a U(\omega) \frac{\omega\left\{\omega^{2}-N^{2}\right\}^{\frac{1}{2}}}{2 N} \ln \frac{\xi-\mathrm{i}}{\xi+\mathrm{i}}, \\
\boldsymbol{v}_{\mathrm{h}}(\boldsymbol{r}, \omega)=U(\omega) \frac{\omega^{2}\left\{\omega^{2}-N^{2}\right\}^{\frac{1}{2}}}{N\left\{\omega^{2}-\Sigma_{+}^{2}\right\}^{\frac{1}{2}}\left\{\omega^{2}-\Sigma_{-2}^{2}\right\}^{\frac{1}{2}}} \frac{\xi}{\xi^{2}+1} \frac{a}{r} \sin \theta \frac{r_{\mathrm{h}}}{r_{\mathrm{h}}} \\
v_{z}(\boldsymbol{r}, \omega)=U(\omega) \frac{\omega^{2}\left\{\omega^{2}-N^{2}\right\}^{\frac{1}{2}}}{N\left\{\omega^{2}-\Sigma_{+}^{2}\right\}^{\frac{1}{2}}\left\{\omega^{2}-\Sigma_{-}^{2}\right\}^{\frac{1}{2}}} \frac{1}{\xi} \frac{a}{r} \cos \theta .
\end{gathered}
$$

Causality extends these results along the whole real $\omega$-axis, by (5.3). Thus, $\xi$ becomes a complex coordinate. Once $U(\omega)$ has been replaced by its value, Fourier inverting (8.11)-(8.14) finally provides the internal waves generated by any pulsation $U(t)$ of the sphere.

In dealing with monochromatic and impulsive pulsations, in what follows we shall consider internal waves at large distances $r \gg a$ from the sphere. There $\Sigma_{ \pm}$approach the frequency $M|\cos \theta|$ of the waves emanating from the origin, according to

$$
\Sigma_{ \pm} \sim N|\cos \theta| \mp N \frac{a}{r} \sin \theta \operatorname{sgn} z
$$




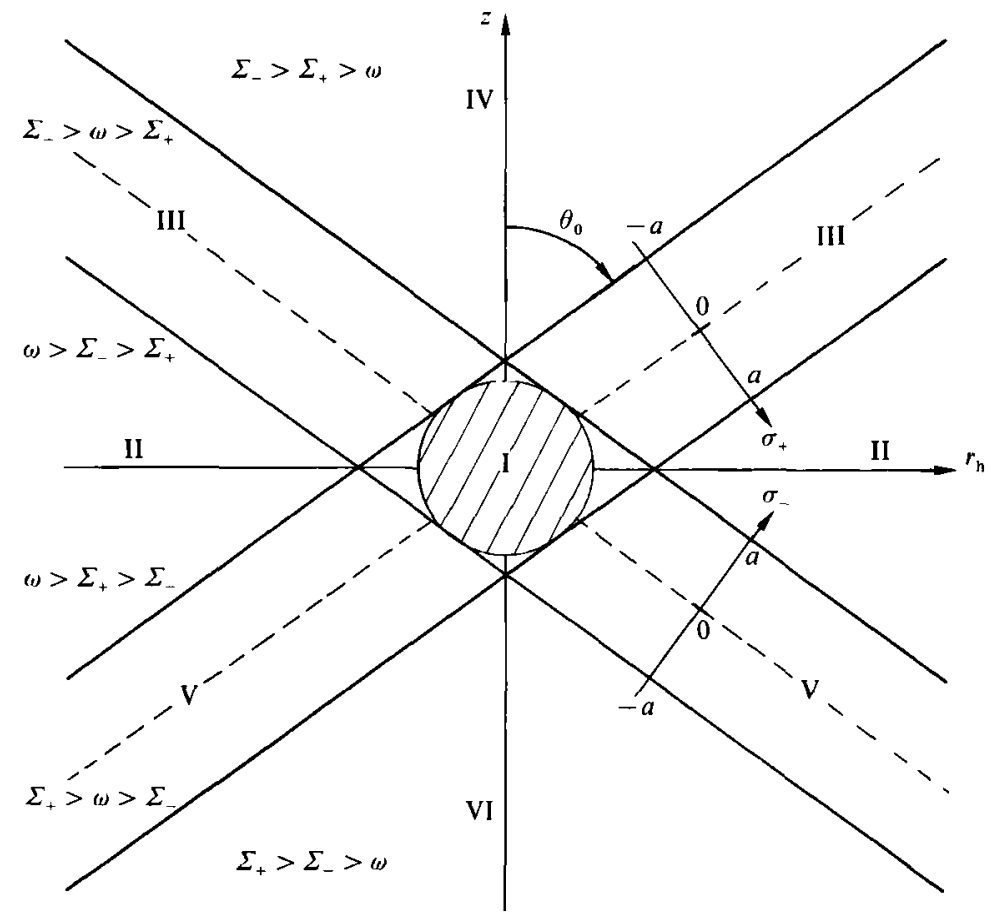

Figure 10. Boussinesq internal wave field of a monochromatically pulsating sphere. Waves are confined in regions III and V, bounded by characteristic cones of semi-angle $\theta_{0}=\arccos (\omega / N)$. There, coordinates $\sigma_{ \pm}$describe transverse distances, with which the phase variates.

and $|\xi| \gg 1$ for almost any frequency. The internal wave field simplifies into

$$
\begin{gathered}
\psi(r, \omega) \sim \frac{a U(\omega)}{N\left\{\omega^{2}-N^{2}\right\}^{\frac{2}{2}} \frac{1}{\xi},} \\
P(\boldsymbol{r}, \omega) \sim \mathrm{i} \rho_{0} a U(\omega) \frac{\omega\left\{\omega^{2}-N^{2}\right\}^{\frac{1}{2}}}{N} \frac{1}{\xi}, \\
v(r, \omega) \sim U(\omega) \frac{\omega^{2}\left\{\omega^{2}-N^{2}\right\}^{\frac{1}{2}}}{N\left\{\omega^{2}-\Sigma_{+}^{2}\right\}^{\frac{1}{2}}\left\{\omega^{2}-\Sigma_{-2}^{2}\right\}^{\frac{1}{2}}} \frac{1}{\xi} \frac{a}{r} \frac{r}{r} .
\end{gathered}
$$

The motion of fluid particles is radial as if, again, the waves emanated from the origin.

\subsection{Monochromatic far field}

The sphere is supposed to pulsate monochromatically, at the frequency $0<\omega<N$. If $U(t)=U_{0} \mathrm{e}^{\mathrm{i} \omega t}$, and the time dependence $\mathrm{e}^{\mathrm{i} \omega t}$ is omitted in all variables, the internal wave field is given by $(8.16)-(8.18)$ with $U(\omega)$ replaced by $U_{0}$. According to the different values of the now complex coordinate $\xi$ the space must be divided into six regions (figure 10), separated by the characteristic cones, of vertical axis and semiangle $\theta_{0}=\arccos (\omega / N)$, tangent to the sphere (Hendershott 1969; Appleby \& Crighton 1987).

In regions II, IV and VI $\xi$ is either real or imaginary, the phase of internal waves does not vary and no energy is radiated. More precisely $\xi$ reduces in the far field $r \gg a$ to

$$
\xi \sim \frac{\left\{\omega^{2}-N^{2} \cos ^{2} \theta\right\}^{\frac{1}{2}}}{N} \frac{r}{a}
$$


so that

$$
\psi(\boldsymbol{r}) \sim \frac{a U_{0}}{\left\{\omega^{2}-N^{2}\right\}^{\frac{1}{2}}\left\{\omega^{2}-N^{2} \cos ^{2} \theta\right\}^{\frac{1}{2}}} \frac{a}{r}=4 \pi a^{2} U_{0} G(\boldsymbol{r}, \omega) .
$$

The pressure and velocity are similarly given by (7.3)-(7.4), with $m_{0}$ replaced by $4 \pi a^{2} U_{0}$. Regions II, IV and VI are those where the point source model is valid; in agreement with $\$ 7.1$ they also are those where no internal waves are found.

On the other hand, in regions III and $\mathrm{V} \xi$ is complex, implying phase variation and energy radiation. Since $|\cos \theta| \rightarrow \omega / N$ as $r / a \rightarrow \infty,(8.19)$ is invalidated. It must be taken into account that transverse distances, represented by Hurley's (1972) characteristic coordinates (cf. figure 10)

$$
\sigma_{ \pm}=r \sin \left(\theta \mp \theta_{0}\right)=\frac{\omega}{N} r_{\mathrm{h}} \mp \frac{\left[N^{2}-\omega^{2}\right]^{\frac{1}{2}}}{N} z,
$$

remain finite and non-zero as the far field is approached. In region III for instance, as $r \gg a>\left|\sigma_{+}\right|$,

so that

$$
\begin{gathered}
r_{\mathrm{h}} \sim \frac{\left[N^{2}-\omega^{2}\right]^{\frac{1}{2}}}{N} r+\frac{\omega}{N} \sigma_{+}, \quad z \sim \frac{\omega}{N} r-\frac{\left[N^{2}-\omega^{2}\right]^{\frac{1}{2}}}{N} \sigma_{+} \\
\xi^{2} \sim \frac{\omega\left[N^{2}-\omega^{2}\right]^{\frac{1}{2}}}{N^{2}} \frac{r}{a} \mathrm{e}^{-\mathrm{i} \arccos \left(\sigma_{+} / a\right)}
\end{gathered}
$$

Then the internal wave field is given by

$$
\begin{gathered}
\psi(r) \sim \mathrm{i} \frac{a U_{0}}{\omega^{\frac{1}{2}}\left[N^{2}-\omega^{2}\right]^{\frac{3}{4}}}\left(\frac{a}{r}\right)^{\frac{1}{2}} \mathrm{e}^{(\mathrm{i} / 2) \arccos \left(\sigma_{+} / a\right)}, \\
P(r) \sim \rho_{0} a U_{0} \omega^{\frac{1}{2}}\left[N^{2}-\omega^{2}\right]^{\frac{1}{4}}\left(\frac{a}{r}\right)^{\frac{1}{2}} \mathrm{e}^{(\mathrm{i} / 2) \arccos \left(\sigma_{+} / a\right)}, \\
v(r) \sim \frac{U_{0}}{2} \frac{\omega^{\frac{1}{2}}}{\left[N^{2}-\omega^{2}\right]^{\frac{1}{4}}}\left(\frac{a}{r}\right)^{\frac{1}{2}} \frac{a}{\left[a^{2}-\sigma_{+}^{2}\right]^{\frac{1}{2}}} \frac{r}{r} \mathrm{e}^{(\mathrm{i} / 2) \arccos \left(\sigma_{+} / a\right)}
\end{gathered}
$$

This expression makes Appleby's \& Crighton's (1987) result for a pulsating sphere more explicit, and is similar to Lighthill's $(1978, \S 4.10)$ result for a distributed mass source.

The phase $\Phi=\omega t+\frac{1}{2} \arccos \left(\sigma_{+} / a\right)$ varies transversely, in agreement with the experiments of McLaren et al. (1973). From its differentiation with respect to $\sigma_{+}$(cf. (7.10)) we deduce the transverse wavelength $\lambda=4 \pi\left(a^{2}-\sigma_{+}^{2}\right)^{\frac{1}{2}}$, and the phase and group velocities

$$
\begin{gathered}
c_{\phi}=\frac{\omega}{k}=2 \omega\left(a^{2}-\sigma_{+}^{2}\right)^{\frac{1}{2}}, \\
c_{\mathrm{g}}=\frac{\left[N^{2}-\omega^{2}\right]^{\frac{1}{2}}}{k}=2\left[N^{2}-\omega^{2}\right]^{\frac{1}{2}}\left(a^{2}-\sigma_{+}^{2}\right)^{\frac{1}{2}},
\end{gathered}
$$

which are zero at the edges $\sigma_{+}= \pm a$ of region III and maxima halfway between them. The definition of $\lambda$ is, however, of purely academic interest, since not even a single oscillation of the phase takes place between these edges. Only a continuous monotonic variation of $\frac{1}{2} \pi$ in $\Phi$ is observed, which replaces the phase jump obtained 
in $\$ 5.1$ between regions II and IV. Interpreting it as a quarter of an oscillation we, rather, as did Appleby \& Crighton (1987), introduce an effective wavelength ' $\lambda$ ' $=8 a$.

The pressure and velocity oscillate in phase and verify, consistently with (4.5),

$$
v \sim \frac{P}{2 \rho_{0}\left[N^{2}-\omega^{2}\right]^{\frac{1}{2}}\left(a^{2}-\sigma_{+}^{2}\right)^{\frac{1}{2}}} \frac{r}{r} .
$$

Their decrease as $1 / r^{\frac{1}{2}}$, consistent with the conservation of energy for conical waves, coincides with the experiments of McLaren et al. (1973) again. The velocity singularity at the edges of region III remains integrable, and the energy flux consequently finite.

\subsection{Impulsive far field}

We now consider an impulsive pulsation $U(t)=U_{0} \delta(t)$ of the sphere, for which $U(\omega)=U_{0}$, and investigate by the asymptotic method of $\S 6.1$ the radiated internal waves. For small times $N t \ll 1$, from the high-frequency expansion of $(8.16)$ $(\xi \sim(\omega / N)(r / a))$ and its Fourier inversion by (D 2), we have

$$
\psi(r, t) \sim-H(t) \frac{a^{2} U_{0}}{r} t \sim 4 \pi a^{2} U_{0} G(r, t) .
$$

Similarly the pressure and velocity are given by (7.5)-(7.6), with $m_{0}$ replaced by $4 \pi a^{2} U_{0}$. As expected the initial motion is irrotational, and the point source model is then valid.

For large times $N t \gg 1$, internal waves separate again into gravity waves (the contribution of the singularities $\pm \Sigma_{+}, \pm \Sigma_{-}$) and buoyancy oscillations (the contribution of the singularities $\pm N$ ). For both of them, although for different reasons, the far-field assumption $r \gg a$ contradicts the use of Lighthill's (1958) method. Thus, we relax it at first. The procedure we shall use instead closely follows that of Bretherton (1967) and Grimshaw (1969).

For gravity waves, as long as $r / a$ remains finite, $\Sigma_{+}$and $\Sigma_{-}$remain separated and Lighthill's method still applies. After some algebra we find in the vicinity of $\Sigma_{ \pm}$, omitting a regular part which makes no contribution to the large-time expansion,

$$
\ln \frac{\xi-\mathrm{i}}{\xi+\mathrm{i}} \sim \mp \mathrm{i}\left(1-\frac{a^{2}}{r^{2}}\right)^{\frac{1}{4}}\left\{\frac{2}{\sin \theta_{ \pm} \sin \theta}\right\}^{\frac{1}{2}}\left\{\frac{\omega-\Sigma_{ \pm}}{\Sigma_{ \pm}}\right\}^{\frac{1}{2}} \operatorname{sgn} \cos \theta_{ \pm} .
$$

Inverting then (8.11) by (D 3) we finally obtain for $r_{\mathrm{h}}>a$

$$
\begin{aligned}
\psi_{\mathrm{g}}(r, t) \sim \frac{H(t)}{(2 \pi)^{\frac{1}{2}}} \frac{a U_{0}}{N}(1- & \left.\frac{a^{2}}{r^{2}}\right)^{\frac{1}{4}} \frac{1}{(\sin \theta)^{\frac{1}{2}}} \\
& \times\left[\frac{\cos \theta_{+}}{\left(\sin \theta_{+}\right)^{\frac{3}{2}}} \frac{\sin \left(\Sigma_{+} t-\frac{1}{4} \pi\right)}{\left(\Sigma_{+} t\right)^{\frac{3}{2}}}-\frac{\cos \theta_{-}}{\left(\sin \theta_{-}\right)^{\frac{3}{2}}} \frac{\sin \left(\Sigma_{-} t-\frac{1}{4} \pi\right)}{\left(\Sigma_{-} t\right)^{\frac{3}{2}}}\right],
\end{aligned}
$$

and for $r_{\mathrm{h}}<a$ with $(z>0, z<0)$

$$
\begin{aligned}
\psi_{\mathrm{g}}(r, t) \sim \frac{H(t)}{(2 \pi)^{\frac{1}{2}}} \frac{a U_{0}}{N}\left(1-\frac{a^{2}}{r^{2}}\right)^{\frac{1}{4}} \frac{1}{(\sin \theta)^{\frac{1}{2}}} \\
\quad \times\left[\frac{\cos \theta_{+}}{\left|\sin \theta_{+}\right|^{\frac{3}{2}}} \frac{(\sin , \cos )\left(\Sigma_{+} t-\frac{1}{4} \pi\right)}{\left(\Sigma_{+} t\right)^{\frac{3}{2}}}-\frac{\cos \theta_{-}}{\left|\sin \theta_{-}\right|^{\frac{3}{2}}} \frac{(\cos , \sin )\left(\Sigma_{-} t-\frac{1}{4} \pi\right)}{\left(\Sigma_{-} t\right)^{\frac{3}{2}}}\right] .
\end{aligned}
$$




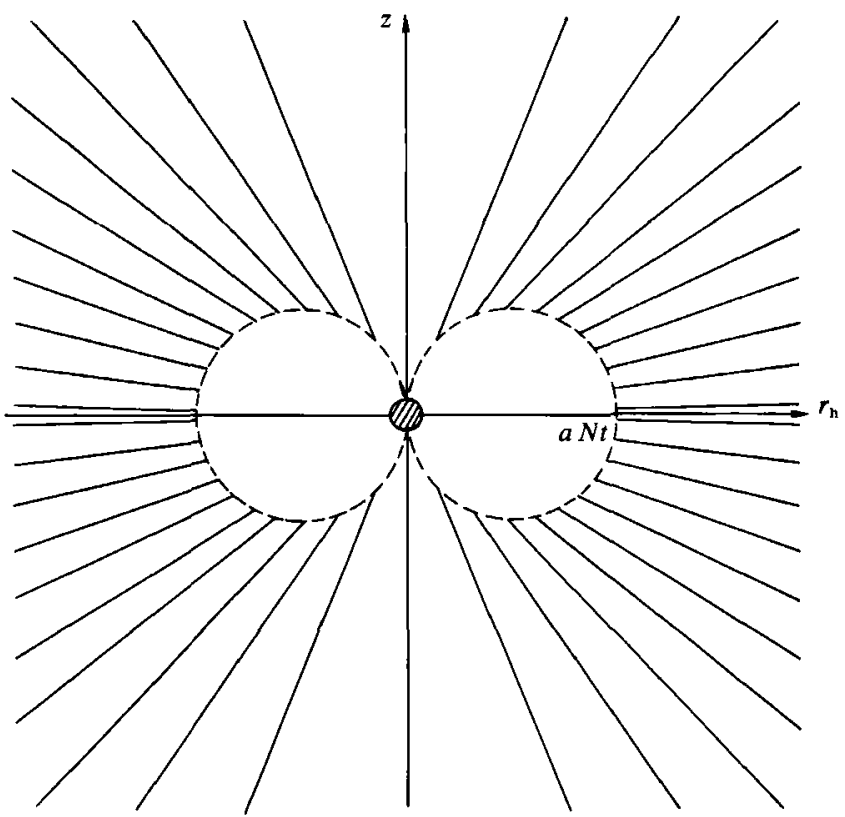

Fiqure 11. Boussinesq internal wave field of an impulsively pulsating sphere. Out of the characteristic torus of radius Nta gravity waves, whose surfaces of constant phase are conical (cf. figure 2), dominate. Inside the torus they give way to buoyancy oscillations, whose phase is constant.

Accordingly the gravity wave field results from the interference between the waves emanating from the points $T_{+}$and $T_{-}$of the sphere.

As the far field $r \gg a$ is entered, the separation between $T_{+}$and $T_{-}$vanishes in the amplitude of these waves but is still present in their phase; there it reduces to the small but fundamental difference (8.15) between $\Sigma_{+}$and $\Sigma_{-}$. Then $(8.32 a)$ becomes

$$
\begin{aligned}
\psi_{\mathrm{g}}(\boldsymbol{r}, t) & \sim-H(t)\left(\frac{2}{\pi}\right)^{\frac{1}{2}} \frac{a U_{0}}{N} \frac{|\cos \theta|}{\sin ^{2} \theta} \sin \left(N t \frac{a}{r} \sin \theta\right) \frac{\cos \left(N t|\cos \theta|-\frac{1}{4} \pi\right)}{(N t|\cos \theta|)^{\frac{3}{2}}}, \\
& \sim 4 \pi a^{2} U_{0} \frac{\sin k_{\mathrm{g}} a}{k_{\mathrm{g}} a} G_{\mathrm{g}}(\boldsymbol{r}, t) .
\end{aligned}
$$

The interference between $T_{+}$and $T_{-}$takes on the familiar form of a factor $\sin \left(k_{\mathrm{g}} a\right) /\left(k_{\mathrm{g}} a\right)$, with $k_{\mathrm{g}}$ the wavevector $(7.10)$, multiplying the gravity waves radiated by a point impulsive source releasing the volume $4 \pi a^{2} U_{0}$. The same is true of the pressure and velocity, which vary as $t^{-\frac{3}{2}}$ and $t^{-\frac{1}{2}}$, respectively, and remain finite at any $t$. Where the sphere is small compared with the wavelength $\lambda_{\mathrm{g}}$ of gravity waves, as

$$
r / a \gg N t \sin \theta
$$

the interference is constructive and the point source is equivalent to the sphere. As for the Boussinesq approximation a torus, hereafter called characteristic, of vertical axis and radius $\mathrm{Nta}$, defines the validity of the point source model for gravity waves.

Note that the distinction between the two forms (8.32) and (8.33) of gravity waves corresponds to the separation of space into two regions similar to those encountered in $\$ 6.2:$ (i) $N t \gg 1$ with $r / t$ fixed, (ii) $N t \gg 1$ with $r / a$ fixed (Bretherton 1967; 
Grimshaw 1969). Equation (8.32) is relevant in region (ii), and (8.33) at the boundary between regions (i) and (ii). A particular feature of the pulsating sphere is that (8.33) remains valid across the whole of region (i); see Voisin (1991).

For buoyancy oscillations $\xi$ vanishes in the vicinity of $N$ for $r_{\mathrm{h}}<a$, according to

$$
\begin{array}{ccc}
\xi & \sim \frac{\left(r_{\mathrm{h}}^{2}-a^{2}\right)^{\frac{1}{2}}}{a} & \left(r_{\mathrm{h}}>a\right), \\
& \sim \sqrt{ } 2 \frac{|z|}{\left(a^{2}-r_{\mathrm{h}}^{2}\right)^{\frac{1}{2}}}\left\{\frac{\omega-N}{N}\right\}^{\frac{1}{2}} & \left(r_{\mathrm{h}}<a\right) .
\end{array}
$$

There (8.16)-(8.18), which rely upon the fact that $|\xi| \gg 1$, no longer describe the far field. Thus we directly apply Lighthill's method to (8.11)-(8.14), use (D 3)-(D 4), and find

$$
\begin{aligned}
& \psi_{\mathrm{b}}(\boldsymbol{r}, t) \sim-H(t)\left(\frac{2}{\pi}\right)^{\frac{1}{2}} \frac{a U_{0}}{N} \arcsin \left(\frac{a}{r_{\mathrm{h}}}\right) \frac{\sin \left(N t-\frac{1}{4} \pi\right)}{(N t)^{\frac{1}{2}}} \quad\left(r_{\mathrm{h}}>a\right),(8.36 a) \\
& \sim-H(t)\left(\frac{\pi}{2}\right)^{\frac{1}{2}} \frac{a U_{0}}{N} \frac{\sin \left(N t-\frac{1}{4} \pi\right)}{(N t)^{\frac{1}{2}}} \quad\left(r_{\mathrm{h}}<a\right),(8.36 b) \\
& P_{\mathrm{b}}(\boldsymbol{r}, t) \sim H(t)\left(\frac{2}{\pi}\right)^{\frac{1}{2}} \rho_{0} N^{2} a U_{0} \arcsin \left(\frac{a}{r_{\mathrm{h}}}\right) \frac{\sin \left(N t-\frac{1}{4} \pi\right)}{(N t)^{\frac{3}{2}}} \quad\left(r_{\mathrm{h}}>a\right),(8.37 a) \\
& \sim H(t)\left(\frac{\pi}{2}\right)^{\frac{1}{2}} \rho_{0} N^{2} a U_{0} \frac{\sin \left(N t-\frac{1}{4} \pi\right)}{(N t)^{\frac{3}{2}}} \quad\left(r_{\mathrm{h}}<a\right),(8.37 b) \\
& \boldsymbol{v}_{\mathrm{b}}(\boldsymbol{r}, t) \sim-H(t)\left(\frac{2}{\pi}\right)^{\frac{1}{2}} N U_{0} \frac{a}{\left(r_{\mathrm{h}}^{2}-a^{2}\right)^{\frac{1}{2}}}\left[\frac{a r_{\mathrm{h}}}{r_{\mathrm{h}}^{2}}, \frac{a z}{r_{\mathrm{h}}^{2}-a^{2}}\right] \frac{\cos \left(N t-\frac{1}{4} \pi\right)}{(N t)^{\frac{3}{2}}} \quad\left(r_{\mathrm{h}}>a\right),(8.38 a) \\
& \sim 0 \\
& \left(r_{\mathrm{h}}<a\right) .(8.38 b)
\end{aligned}
$$

For $r_{\mathrm{h}}<a$ the velocity field is made zero by the regularity of $(8.13)-(8.14)$ near $N$. For $r_{\mathrm{h}}>a$ the two terms in square brackets in $(8.38 a)$ respectively denote its horizontal and vertical components.

Inside the vertical cylinder circumscribing the sphere, buoyancy oscillations induce no motion of the fluid. On the cylinder the velocity diverges. Outside it the way that the finite extent of the sphere modifies buoyancy oscillations is different for $\left(\psi_{\mathrm{b}}, P_{\mathrm{b}}\right)$ on the one hand, and $v_{\mathrm{b}}$ on the other hand. For $\psi_{\mathrm{b}}$ for instance,

$$
\psi_{\mathrm{b}}(r, t) \sim 4 \pi a^{2} U_{0} \frac{r_{\mathrm{h}}}{a} \arcsin \left(\frac{a}{r_{\mathrm{h}}}\right) G_{\mathrm{b}}(r, t) .
$$

In both cases, however, very far from the cylinder, as

$$
r_{\mathrm{h}} \gg a
$$

the point impulsive source releasing a volume $4 \pi a^{2} U_{0}$ is again equivalent to the sphere. Although this criterion is consistent with the cylindrical nature of the surfaces where the wavelength (7.20) of buoyancy oscillations is constant, the explanation for its exact form does not involve this wavelength; see Voisin (1991).

The structure of impulsive internal waves, generated by the sphere, consequently evolves as follows (figure 11), in the far field $r \gg a$ and for large times $N t \gg 1$. At first, for a fixed observer, the sphere behaves like a point and the internal wave field is 
dominated by gravity waves. The surfaces of constant phase are conical and the wavelength decreases as $t^{-1}$; the pressure decays as $t^{-\frac{1}{2}}$ while the velocity grows as $t^{\frac{1}{2}}$. Next the characteristic torus, which expands along the horizontal at the velocity $\mathrm{Na}$, reaches the point under consideration. The wavelength becomes comparable with the radius of the sphere, and gravity waves are 'blurred' by the interference between the points $T_{+}$and $T_{-}$. Now both pressure and velocity decay, as $t^{-\frac{3}{2}}$ and $t^{-\frac{1}{2}}$ respectively. So buoyancy oscillations, for which they vary as $t^{-\frac{3}{2}}$, become of the same order as gravity waves. Further inside the torus interference turns out to be destructive and gravity waves vanish. There only remain buoyancy oscillations, whose structure is ruled by the vertical cylinder circumscribing the sphere. Far from it, the point source model is valid again; near to it, 'interference' of a special kind arises; inside it, the fluid stays at rest.

The experiments of Stevenson (1973) for an impulsive source and McLaren et al. (1973) for a transient source both confirm this discussion. The former exhibited the disappearance of gravity waves inside an expanding surface of roughly toroidal shape, and the latter showed the ultimate dominance of oscillations of the fluid at the buoyancy frequency. Similarly, an ultimate passage from gravity waves to buoyancy oscillations has been shown by the theoretical studies of Sekerzh-Zen'kovich (1982) and Chashechkin \& Makarov (1984).

\section{Conclusion}

In this paper a complete examination of the Green's function of internal gravity waves has been attempted. For both monochromatic and impulsive waves, in both Boussinesq and non-Boussinesq cases, the Green's function has been calculated. From it the pressure and velocity fields radiated by a point source have been deduced; parallel to it the Boussinesq internal waves generated by a pulsating sphere have been derived. In so doing not only a first, mainly mathematical, aim has been achieved, namely to synthesize and complement previous fragmentary and sometimes contradictory results about the Green's function; but also a second, mainly physical, aim has been reached, namely to analyse three interrelated principles which appear to rule the generation of internal waves: the dual structure of time-dependent internal wave fields, which combine gravity and buoyancy waves, the validity of the Boussinesq approximation, and the validity of the point source model.

To deal with both the Green's function and the pulsating sphere, the method we used is the same and applies to any problem of internal wave radiation. Monochromatic waves are considered first, by a complex coordinate transformation which reduces the internal wave equation to a Helmholtz equation; causality makes the transformation determinate. Then impulsive waves are investigated in the smalland large-time limits, in which cases they follow from the expansions of monochromatic waves near high and singular frequencies, respectively, and their Fourier inversion with respect to time.

At a fixed frequency $\omega<N$, the Green's function satisfactorily describes nonBoussinesq internal waves, propagating outside and evanescent inside the characteristic cone $|\cos \theta|=\omega / N, \theta$ denoting the observation angle from the upward vertical. Under the Boussinesq approximation, on the other hand, the Green's function is consistent with the confinement of the waves on this cone but fails to represent them there; thus, the point source model must be given up. Replacing it by a sphere gives the cone a width equal to the diameter of the sphere; in this conical 


\begin{tabular}{|c|c|c|c|}
\hline Type of wave & Acoustic & Electromagnetic & Gravity \\
\hline Small source & $a \ll \lambda$ & $a \ll \lambda$ & $\begin{array}{l}\qquad r \gg N t a \sin \theta \\
\quad>\text { outside the } \\
\text { characteristic torus }\end{array}$ \\
\hline Near zone & $\begin{array}{c}a \ll r \ll \lambda \\
\rightarrow \text { incompressible fluid }\end{array}$ & $\begin{aligned} & a \ll r \ll \lambda \\
& \rightarrow \text { static fields }\end{aligned}$ & $\begin{array}{c}N t \ll 1 \text { and } r \gg a \\
\rightarrow \text { homogeneous fluid }\end{array}$ \\
\hline Far zone & $\begin{array}{c}\quad a \ll \lambda \ll r \\
\rightarrow \text { radiation of } \\
\text { acoustic waves }\end{array}$ & $\begin{array}{c}\quad a \ll \lambda \ll r \\
\rightarrow \text { radiation of } \\
\text { electromagnetic waves }\end{array}$ & $\begin{aligned} N t \gg & 1 \text { and } r \gg N t a \sin \theta \\
& \rightarrow \text { radiation of } \\
& \text { gravity waves }\end{aligned}$ \\
\hline
\end{tabular}

TABLE 1. Compared structures of classical wave fields and Boussinesq gravity wave fields. The characteristics of the latter are attributable to their wavelength $\lambda_{\mathrm{g}}=(2 \pi / N t)(r / \sin \theta)$.

shell an explicit expression for the waves is found, which accounts for their $1 / r^{\frac{1}{2}}$ radial decay, and their transverse phase variations.

For large times $N t \gg 1$ after an impulse, internal waves split into gravity and buoyancy waves; the splitting follows the arrival of an Airy wave and is attributable to non-Boussinesq effects. As time elapses, the two components increasingly separate and ultimately lose, even if only superficially, their non-Boussinesq nature. Boussinesq gravity waves are plane propagating internal waves of frequency $N|\cos \theta|$, whose wavelength

$$
\lambda_{\mathrm{g}}=\frac{2 \pi}{N t} \frac{r}{\sin \theta}
$$

is constant on tori. Boussinesq buoyancy waves are radial oscillations at frequency $N$, found everywhere in the fluid; in fact they are propagating waves, of horizontal wavevector and wavelength

$$
\lambda_{\mathrm{b}}=\frac{4 \pi}{\beta}\left(\frac{\beta r_{\mathrm{h}}}{2 N t}\right)^{\frac{1}{3}}
$$

constant on cylinders $\left(r_{\mathrm{h}}\right.$ denotes the horizontal distance from the source and $2 / \beta$ the scale height of the stratification), whose propagation has vanished as they have become Boussinesq.

For both gravity and buoyancy waves the Boussinesq approximation implies small wavelengths $\lambda \ll 2 / \beta$. Thus it is valid, for the former when

$$
\frac{1}{2} \beta r \ll N t \sin \theta \text {, }
$$

and for the latter when

$$
\frac{1}{2} \beta r_{\mathrm{h}} \ll N t,
$$

that is inside a torus of vertical axis and radius $2 N t / \beta$, and inside the circumscribing cylinder, respectively (figure 8). As these regions are entered, buoyancy waves, initially comparable with gravity waves, simultaneously stop propagating and become less significant.

There two separate criteria define, again, the validity of the point source model; for a spherical source of radius $a \ll 2 / \beta$ they are

$$
r / a \gg N t \sin \theta
$$

for gravity waves, and

$$
r_{\mathrm{h}} \gg a
$$

for buoyancy oscillations. Outside the torus of vertical axis and radius Nta, gravity 
waves are dominant (figure 11); for them the sphere is compact, since $a \ll \lambda_{\mathrm{g}}$. Inside this torus interference caused by the finite size of the sphere blurs gravity waves, and buoyancy oscillations are observed. For them the sphere is compact far from the vertical cylinder circumscribing it. Inside this cylinder the fluid is quiescent.

Let us finally emphasize that, however different from the fields of acoustic or electromagnetic waves internal wave fields may be, their structure is governed by the same underlying principles, with the amendments required by the special form of the wavelengths (9.1) and (9.2) of their two components. To illustrate this fact table 1 compares the different zones of a Boussinesq gravity wave field with those of acoustic and electromagnetic wave fields (cf. Jackson 1975, §9.1 and Pierce 1981, §4.7).

This work is part of a Ph.D. thesis prepared at Université Pierre et Marie Curie and Thomson-Sintra ASM. It has been written during a one-year stay at the Institut de Mécanique de Grenoble, whose hospitality is acknowledged. The author would like to thank Professor Zarembowitch of UPMC, and Dr Tran Van Nhieu of ThomsonSintra, for their guidance throughout his Ph.D.; he is also indebted to Professors Crighton and Hopfinger for helpful discussions and kind encouragement given at decisive steps of the study. Financial support by the ANRT through Convention CIFRE No. 249/87 is acknowledged.

\section{Appendix A. Internal wave generation by point mass and force sources}

When a force source $F$ per unit volume is added to the mass source considered in $\$ 3.1$, the horizontal motion of the fluid may become rotational. No internal potential exists any more, and a direct derivation of the equations verified by the physical variables $v, P$ and $\rho$ must be performed. In terms of the vertical displacement $\zeta_{z}$ and the pressure $P$, eliminating $v_{\mathrm{h}}$ and $\rho$ from (3.2)-(3.4), then removing the density factors (3.9), we obtain

$$
\begin{gathered}
\mathrm{L} \zeta_{z}=\left(\frac{\partial}{\partial z}-\frac{\beta}{2}\right) \frac{\partial}{\partial t}\left(\mathrm{e}^{-\beta z / 2} m\right)-\frac{1}{\rho_{00}} \boldsymbol{e}_{z} \cdot \nabla \times\left[\left(\nabla-\frac{1}{2} \beta \boldsymbol{e}_{z}\right) \times\left(\mathrm{e}^{\beta z / 2} \boldsymbol{F}\right)\right] \\
\mathrm{L} P=-\rho_{00}\left(\frac{\partial^{2}}{\partial t^{2}}+N^{2}\right) \frac{\partial}{\partial t}\left(\mathrm{e}^{-\beta z / 2} m\right)+\left[\frac{\partial^{2}}{\partial t^{2}}\left(\nabla+\frac{1}{2} \beta \boldsymbol{e}_{z}\right)+N^{2} \nabla_{\mathrm{h}}\right] \cdot\left(\mathrm{e}^{\beta z / 2} \boldsymbol{F}\right)
\end{gathered}
$$

where

$$
\mathrm{L}=\frac{\partial^{2}}{\partial t^{2}}\left(\nabla^{2}-\frac{1}{4} \beta^{2}\right)+N^{2} \nabla_{\mathrm{h}}^{2}
$$

From these equations, and the definition (3.14) of the Green's function $G(r, t)$, the internal wave field generated by the point mass and force sources $m(\boldsymbol{r}, t)=m_{0} \delta(\boldsymbol{r}) \delta(t)$ and $F(r, t)=F_{0} \delta(r) \delta(t)$ readily follows as

$$
\begin{gathered}
\zeta_{z}(\boldsymbol{r}, t)=\left\{m_{0}\left(\frac{\partial}{\partial z}-\frac{\beta}{2}\right) \frac{\partial}{\partial t}-\frac{1}{\rho_{00}}\left[F_{0_{\mathrm{h}}}\left(\frac{\partial}{\partial z}-\frac{\beta}{2}\right)-F_{0_{z}} \nabla_{\mathrm{h}}\right] \cdot \nabla_{\mathrm{h}}\right\} G(\boldsymbol{r}, t), \\
P(\boldsymbol{r}, t)=\left\{-\rho_{00} m_{0}\left(\frac{\partial^{2}}{\partial t^{2}}+N^{2}\right) \frac{\partial}{\partial t}+\boldsymbol{F}_{0} \cdot\left[\frac{\partial^{2}}{\partial t^{2}}\left(\boldsymbol{\nabla}+\frac{1}{2} \beta \boldsymbol{e}_{z}\right)+N^{2} \nabla_{\mathrm{h}}\right]\right\} G(\boldsymbol{r}, t) .
\end{gathered}
$$

For the point mass source $P(r, \omega)$ has already been calculated in $(7.1)$, while $\zeta_{z}(r, \omega)$ 
reduces to $-\mathrm{i} / \omega$ times the vertical component of the velocity (7.2). Closely analogous are the pressure

$$
\begin{aligned}
P(r, \omega)=\frac{F_{0_{z}}}{4 \pi r^{2}} \omega^{2} \frac{\left\{\omega^{2}-N^{2}\right\}^{\frac{1}{2}}}{\left\{\omega^{2}-N^{2} \cos ^{2} \theta\right\}^{\frac{3}{2}}} \exp \left(-\frac{\beta r}{2}\left\{\frac{\omega^{2}-N^{2} \cos ^{2} \theta}{\omega^{2}-N^{2}}\right\}^{\frac{1}{2}}\right) \\
\times\left\{\left[1+\frac{\beta r}{2}\left\{\frac{\omega^{2}-N^{2} \cos ^{2} \theta}{\omega^{2}-N^{2}}\right\}^{\frac{1}{2}}\right] \cos \theta-\frac{\beta r}{2} \frac{\omega^{2}-N^{2} \cos ^{2} \theta}{\omega^{2}-N^{2}}\right\},
\end{aligned}
$$

and the vertical displacement (in the non-Boussinesq far field $\frac{1}{2} \beta r \gg 1$ )

$$
\zeta_{z}(r, \omega) \sim \frac{F_{0_{z}}}{4 \pi \rho_{00} r^{3}} \frac{\beta^{2} r^{2}}{4} \frac{\omega^{4} \sin ^{2} \theta}{\left\{\omega^{2}-N^{2}\right\}^{\frac{3}{2}}\left\{\omega^{2}-N^{2} \cos ^{2} \theta\right\}^{\frac{3}{2}}} \exp \left(-\frac{\beta r}{2}\left\{\frac{\omega^{2}-N^{2} \cos ^{2} \theta}{\omega^{2}-N^{2}}\right\}^{\frac{1}{2}}\right),
$$

generated by a vertical point force source.

Sarma \& Naidu (1972a), Ramachandra Rao (1973) and Grigor'ev \& Dokuchaev (1970) considered the pressure field, and Rehm \& Radt (1975) the Boussinesq vertical displacement, radiated by a monochromatic point mass source. Then Sarma \& Naidu $(1972 b)$ and Ramachandra Rao (1975) obtained the pressure, and Tolstoy (1973, $\S 7.3)$ the far-field non-Boussinesq vertical displacement, generated by a vertical point force source. Of all these calculations only those of Sarma \& Naidu $(1972 a, b)$, Ramachandra Rao (1975) and Tolstoy (1973) disagree with ours, the discrepancies being extra factors $-\frac{1}{2}$ if $|\omega|>N$ and $+\frac{1}{2}$ if $|\omega|<N$ for Ramachandra Rao, $-\frac{1}{2}$ for Tolstoy. The 1975 result of Ramachandra Rao is, moreover, not consistent with his 1973 one, while the pressure of Sarma \& Naidu never propagates. In $\$ 5.1$ an explanation for these differences is given.

\section{Appendix B. Initial conditions for Boussinesq internal waves}

According to the discussion by Batchelor $(1967, \S 6.10)$ of impulsively started motions, body forces and inertial terms are initially negligible compared with the pressure gradients and acceleration of the fluid. Thus, during that stage, a Boussinesq rotating stratified fluid ignores both rotation and stratification. Its motion is irrotational, for the Euler equation becomes

$$
\frac{\partial \boldsymbol{v}}{\partial t}=-\boldsymbol{\nabla} \frac{P}{\rho_{0}}
$$

Closely related with this reasoning is the controversy which, forty years ago, arose about the question of initial conditions for rotating or stratified fluids. Some authors (such as Stewartson 1952) take as initial the state of rest of the fluid; others (such as Morgan 1953) claim the only valid initial conditions to be the irrotational motion (B 1). The second approach, although it may involve somewhat lengthy calculations, remains the more widely used one. In fact, the controversy is but a matter of terminology: zero initial data correspond to $t=0_{-}$, just before the start of the motion, and irrotational initial data to $t=0_{+}$, just after the motion has begun. Any approach which is based upon generalized function theory naturally incorporates the discontinuity at $t=0$ and, whatever initial conditions are chosen, yields the same motion for the fluid, for $t<0$ causal and for $t>0$ initially irrotational.

This we noticed in dealing with the Green's function and can prove in a more formal way by considering Sekerzh-Zen'kovich's (1982) solution of the Cauchy 
problem for Boussinesq internal waves. For a causal mass source $m(\boldsymbol{r}, t)$, and initial data

$$
\psi_{0}(\boldsymbol{r})=\left.\psi\right|_{t-0}, \quad \psi_{\mathbf{1}}(\boldsymbol{r})=\left.\frac{\partial \psi}{\partial t}\right|_{t=0},
$$

the radiated internal waves (expressed in terms of the internal potential) are given by

$$
\begin{aligned}
\psi(\boldsymbol{r}, t)=\int_{0}^{t} \mathrm{~d} \tau \int \mathrm{d}^{3} \boldsymbol{r}^{\prime} m\left(\boldsymbol{r}^{\prime}, \tau\right) G\left(\boldsymbol{r}-\boldsymbol{r}^{\prime}, t-\tau\right) \\
+\int \mathrm{d}^{3} \boldsymbol{r}^{\prime}\left[\nabla^{2 \prime} \psi_{\mathbf{1}}\left(\boldsymbol{r}^{\prime}\right) G\left(\boldsymbol{r}-\boldsymbol{r}^{\prime}, t\right)+\nabla^{2 \prime} \psi_{0}\left(\boldsymbol{r}^{\prime}\right) \frac{\partial G}{\partial t}\left(\boldsymbol{r}-\boldsymbol{r}^{\prime}, t\right)\right]
\end{aligned}
$$

For a point impulsive source releasing a unit volume of fluid, the internal potential reduces to the Green's function. Now, if the initial state of the fluid is taken just before the impulse, at $t=0_{-}, \psi_{0}(r)=0, \psi_{1}(r)=0, m(r, t)=\delta(r) \delta(t)$ and $G(r, t)$ arises from the mass source term in (B 3). If, on the other hand, the initial state refers to $t=0_{+}$, just after the impulse, irrotational initial conditions are $\psi_{0}(r)=0$ and $\psi_{1}(r)=-1 /(4 \pi r)$, while $m(r, t)=0 ; G(r, t)$ arises now from the initial data term in (B 3), by virtue of $\nabla^{2}(1 / r)=-4 \pi \delta(r)$. Thus, the equivalence of both approaches is proved for the Green's function. As any internal wave field may be built by a superposition of elementary impulses, this conclusion is at the same time proved from a general point of view.

\section{Appendix C. Another kind of asymptotic expansion}

Alternative large-time expansions of the Green's function follow from expanding $G(r, \omega)$, in the vicinity of singularities such as $\pm \omega_{0}$, in terms of $\left\{\omega^{2}-\omega_{0}^{2}\right\}^{\frac{1}{2}}$ instead of $\left\{\omega-\omega_{0}\right\}^{\frac{1}{2}}$. A necessary condition for the validity of this procedure is, however, that $G(r, \omega)$ have the same expansion near opposite frequencies. This is not a trivial restriction since, according to (5.3), the square roots involved are not even functions of frequency.

In this way we obtain for the Boussinesq Green's function, applying (D 6) to (6.1),

$$
\begin{gathered}
G_{\mathrm{B}_{\mathrm{g}}}(\boldsymbol{r}, t) \sim-\frac{H(t)}{4 \pi N r \sin \theta} J_{0}(N t|\cos \theta|), \\
\frac{\partial G_{\mathrm{B}_{\mathrm{b}}}}{\partial t}(\boldsymbol{r}, t) \sim-\frac{H(t)}{4 \pi r \sin \theta} J_{0}(N t) .
\end{gathered}
$$

To leading order in $(N t)^{-\frac{1}{2}},(6.15)$ and $(\mathrm{C} 1)-(\mathrm{C} \mathrm{2})$ of course coincide. Hendershott (1969) derived, for the transient gravity waves generated by a pulsating sphere (equation (39) of his paper), an expansion of this type. To leading order his result (with the appropriate slight modifications), upon which he bases his investigations of monochromatic internal waves, differs from (8.33a). In particular when $a \ll \lambda_{\mathrm{g}}$ it does not reduce to the point source result $(6.15)$. This seems to be attributable to the omission of the above condition.

In the non-Boussinesq case we obtain for gravity waves, applying (D 7) to (5.2),

$$
G_{\mathrm{g}}(r, t) \sim-\frac{H\left(N t-\frac{\beta r}{2 \sin \theta}\right)}{4 \pi N r \sin \theta} J_{0}\left[\left(N^{2} t^{2}-\frac{\beta^{2} r^{2}}{4 \sin ^{2} \theta}\right)^{\frac{1}{2}}|\cos \theta|\right] .
$$


To leading order, as $N t \gg 1$ with $\beta r$ fixed, (C 3) coincides with (6.20); it reduces, when $\theta \approx \frac{1}{2} \pi$, to Dickinson's (1969) formula (76). For gravity waves the non-Boussinesq internal wave front may consequently be approximated by the torus $\frac{1}{2} \beta r=N t \sin \theta$ which also defines, according to $(6.22)$, the region of validity of the Boussinesq approximation.

\section{Appendix D. Some inverse Fourier transforms}

In this paper the following Fourier transforms $F(\omega)$ and original functions $f(t)$ have been used:

$$
\begin{array}{cc}
F(\omega) & f(t) \\
\omega^{n} & \mathrm{e}^{-\mathrm{i} n \pi / 2} \delta^{(n)}(t) \\
\frac{1}{\omega^{n}} \quad(n \neq 0) & H(t) \frac{t^{n-1}}{(n-1) !} \mathrm{e}^{\mathrm{i} n \pi / 2} \\
\omega^{\alpha} \quad(\alpha \text { non-integer }) & -H(t) \frac{\sin \alpha \pi}{\pi} \frac{\Gamma(\alpha+1)}{t^{\alpha+1}} \mathrm{e}^{-\mathrm{i} \alpha \pi / 2} \\
\frac{1}{\omega^{\alpha}} & H(t) \frac{t^{\alpha-1}}{\Gamma(\alpha)} \mathrm{e}^{\mathrm{i} \alpha \pi / 2} \\
\frac{1}{\omega^{2}-\omega_{0}^{2}} & -H(t) \frac{\sin \omega_{0} t}{\omega_{0}} \\
\frac{\exp \left[-\mathrm{i} t_{0}\left\{\omega^{2}-\omega_{0}^{2}\right\}^{\frac{1}{2}}\right]}{\left\{\omega^{2}-\omega_{0}^{2}\right\}^{\frac{1}{2}}} & \mathrm{i} H(t) J_{0}\left(\omega_{0} t\right) \\
\frac{\exp \left(-\mathrm{i} \alpha \omega^{\frac{1}{2}}\right)}{\omega^{\frac{1}{2}}} & H\left(t-t_{0}\right) J_{0}\left[\omega_{0}\left(t^{2}-t_{0}^{2}\right)^{\frac{1}{2}}\right] \\
\frac{\exp \left(-\alpha \omega^{-\frac{1}{2}}\right)}{\omega^{\frac{1}{2}}} & H(t) \frac{\mathrm{e}^{\mathrm{i} \pi / 4}}{(\pi t)^{\frac{1}{2}}} \sum_{n=0}^{\infty} \frac{\mathrm{e}^{-3 i n \pi / 4}}{n !} \frac{\pi^{\frac{1}{2}}}{\Gamma\left(\frac{n+1}{2}\right)}\left(\alpha^{2} t\right)^{n / 2}
\end{array}
$$

Here $n$ represents a non-negative integer and $\alpha, \omega_{0}$ and $t_{0}$ positive real numbers.

In accordance with (3.16)-(3.17), Fourier transforms are defined by

$$
\begin{gathered}
F(\omega)=\int f(t) \mathrm{e}^{-\mathrm{i} \omega t} \mathrm{~d} t \equiv \mathrm{FT}[f(t)], \\
f(t)=\frac{1}{2 \pi} \psi F(\omega) \mathrm{e}^{\mathrm{i} \omega t} \mathrm{~d} \omega \equiv \mathrm{FT}^{-1}[F(\omega)] .
\end{gathered}
$$

They are related to Laplace transforms by

$$
f(t)=\mathrm{FT}^{-1}[\boldsymbol{F}(\omega)]=\mathrm{LT}^{-1}[F(-\mathrm{i} s)]
$$

where $s$ denotes the complex variable involved in Laplace transforms.

Three methods, described in greater detail by Voisin (1991), have been used to derive formulae (D 1)-(D 9): reproduction from Lighthill's (1958, p. 43) table of 


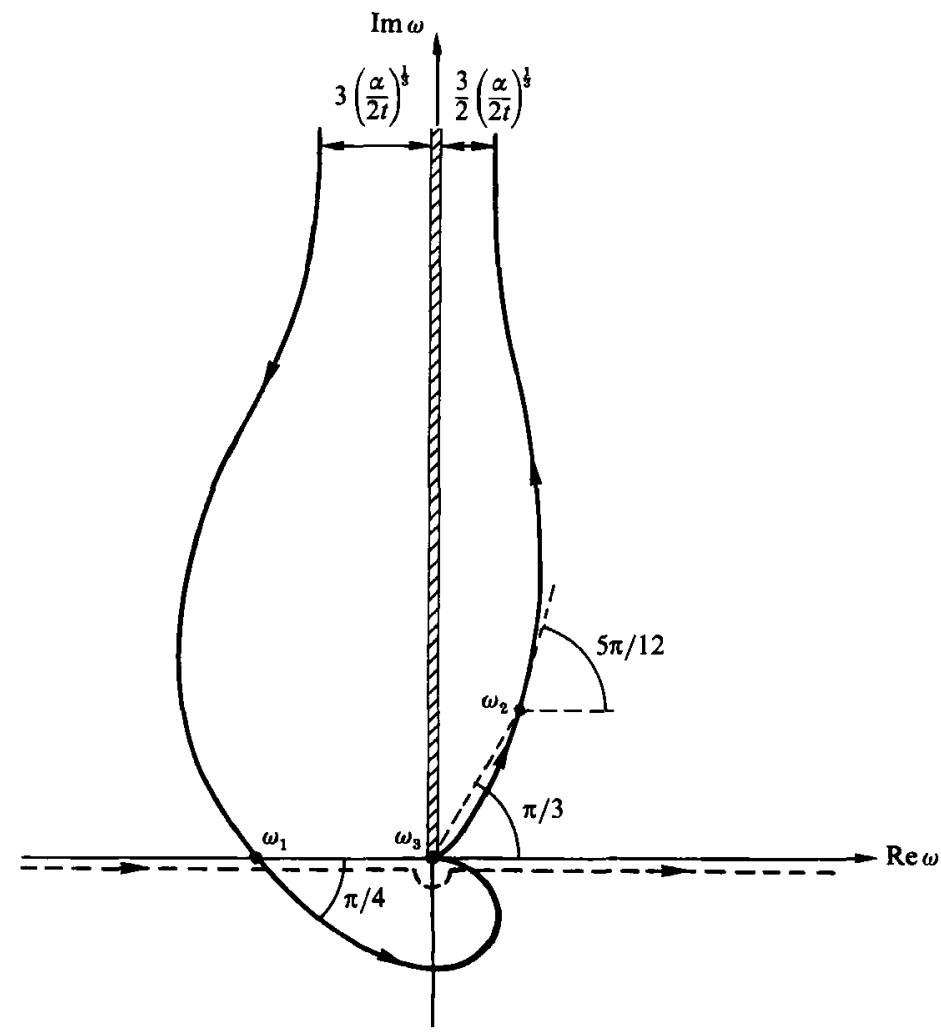

FiaUre 12. Original integration path (dotted line), and steepest descent contours (heavy lines), for the asymptotic expansion of (D 12). The saddle points $\omega_{1}$ and $\omega_{2}$ are situated a distance $(\alpha / 2 t)^{\frac{2}{3}}$ from the origin $\omega_{3}=0$ which makes no contribution to the expansion.

Fourier transforms, application of the residue theorem, and combination of various integrals given by Gradshteyn \& Ryzhik (1980) and Abramowitz \& Stegun (1965). Equation (D 2) differs from Lighthill's corresponding result, defined as a principal value. Most of the inverse Fourier transforms may also be derived from the tables of inverse Laplace transforms of Abramowitz \& Stegun (1965, ch. 29) or Dickinson (1969), by applying (D 11).

The exact inverse transform (D 9), a power series of $t^{\frac{1}{2}}$, is of little interest as a largetime expansion. So, rather, we evaluate it asymptotically, by the method of steepest descents (cf. Bleistein 1984, ch. 7). Consider more generally

$$
f(t)=\mathrm{FT}^{-1}\left[\frac{\exp \left(-\alpha \omega^{-\frac{1}{2}}\right)}{\omega^{8}}\right]=\frac{1}{2 \pi} \psi^{\mathrm{e}^{\mathrm{t} \phi(\omega)}} \frac{\omega^{8}}{\mathrm{~d} \omega}
$$

where

$$
\phi(\omega)=\mathrm{i} \omega-\frac{\alpha}{t} \omega^{-\frac{1}{2}}
$$

Among the three critical points of the integrand,

$$
\omega_{1}=\left(\frac{\alpha}{2 t}\right)^{\frac{2}{3}} \mathrm{e}^{-\mathrm{i} \pi}, \quad \omega_{2}=\left(\frac{\alpha}{2 t}\right)^{\frac{2}{3}} \mathrm{e}^{1 \pi / 3}, \quad \omega_{3}=0,
$$

only the two saddle points $\omega_{1}$ and $\omega_{2}$ contribute to the asymptotic expansion. The paths of steepest descent through them stretch out from zero to infinity along the 
positive imaginary axis (figure 12). Once the original integration path has been deformed, we find

$$
f(t) \sim H(t)\left(\frac{\alpha}{2 t}\right)^{\frac{1-2 s}{3}} \frac{\exp \left[-\frac{3}{2} \mathrm{i}\left(2 \alpha^{2} t\right)^{\frac{1}{3}}-\mathrm{i} \frac{1-4 s}{4} \pi\right]+\exp \left[-\frac{3}{2}\left(2 \alpha^{2} t\right)^{\frac{1}{3}} \mathrm{e}^{-\mathrm{i} \pi / 6}+\mathrm{i} \frac{5-4 s}{12} \pi\right]}{(3 \pi t)^{\frac{1}{2}}} .
$$

\section{REFERENCES}

Abramowitz, M. \& Stegun, I. A. (eds.) 1965 Handbook of Mathematical Functions. Dover.

Appleby, J. C. \& CRighton, D. G. 1986 Non-Boussinesq effects in the diffraction of internal waves from an oscillating cylinder. Q. J. Mech. Appl. Maths 39, 209-231.

Appleby, J. C. \& CRighton, D. G. 1987 Internal gravity waves generated by oscillations of a sphere. J. Fluid Mech. 183, 439-450.

Baines, P. G. 1971 The reflexion of internal/inertial waves from bumpy surfaces. J. Fluid Mech. 46, 273-291.

Barcilon, V. \& Bleistein, N. 1969 Scattering of inertial waves in a rotating fluid. Stud. Appl. Maths 48, 91-104.

Batchelor, G. K. 1967 An Introduction to Fluid Dynamics. Cambridge University Press.

Bleistein, N. 1984 Mathematical Methods for Wave Phenomena. Academic.

Bleistein, N. \& Handelsman, R. A. 1986 Asymptotic Expansions of Integrals. Dover.

Brekhovskikh, L. \& Goncharov, V. 1985 Mechanics of Continua and Wave Dynamics. Springer.

Bretherton, F. P. 1967 The time-dependent motion due to a cylinder moving in an unbounded rotating or stratified fluid. J. Fluid Mech. 28, 545-570.

Chashechkin, Yu. D. \& Makarov, S. A. 1984 Time-varying internal waves. Dokl. Earth Sci. Sect. 276, 210-213.

Cole, J. D. \& Greifinger, C. 1969 Acoustic-gravity waves from an energy source at the ground in an isothermal atmosphere. J. Geophys. Res. 74, 3693-3703.

Dickinson, R. E. 1969 Propagators of atmospheric motions. 1. Excitation by point impulses. Rev. Geophys. 7, 483-514.

Felsen, L. B. 1969 Transients in dispersive media, Part I: Theory. IEEE Trans. Antennas Propag. 17, 191-200.

Gordon, D. \& Stevenson, T. N. 1972 Viscous effects in a vertically propagating internal wave. J. Fluid Mech. 56, 629-639.

Gorodtsov, V. A. \& Teodorovich, E. V. 1980 On the generation of internal waves in the presence of uniform straight-line motion of local and nonlocal sources. Izv. Atmos. Ocean. Phys. 16, 699-704.

Gorodtsov, V.A. \& Teodorovich, E. V. 1983 Radiation of internal waves by periodically moving sources. J. Appl. Mech. Tech. Phys. 24, 521-526.

Gradshteyn, I. S. \& Ryzhik, I. M. 1980 Table of Integrals, Series, and Products (Corrected and Enlarged Edition). Academic.

Grigor'ev, G. I. \& Dokuchaev, V. P. 1970 On the theory of the radiation of acoustic-gravity waves by mass sources in a stratified isothermal atmosphere. Izv. Atmos. Ocean. Phys. 6, 398-402.

Grimshaw, R. 1969 Slow time-dependent motion of a hemisphere in a stratified fluid. Mathematika 16, 231-248.

HART, R. W. 1981 Generalized scalar potentials for linearized three-dimensional flows with vorticity. Phys. Fluids 24, 1418-1420.

Hendershott, M. C. 1969 Impulsively started oscillations in a rotating stratified fluid. J. Fluid Mech. 36, 513-527.

HuRLeY, D. G. 1972 A general method for solving steady-state internal gravity wave problems. J. Fluid Mech. 56, 721-740. 
JACkson, J. D. 1975 Classical Electrodynamics (2nd edn). Wiley.

Kamachi, M. \& HonJi, H. 1988 Interaction of interfacial and internal waves. Fluid Dyn. Res. 2, $229-241$.

KATo, S. $1966 a$ The response of an unbounded atmosphere to point disturbances. I. Timeharmonic disturbances. Astrophys. J. 143, 893-903.

KaTo, S. $1966 b$ The response of an unbounded atmosphere to point disturbances. II. Impulsive disturbances. Astrophys. J. 144, 326-336.

Krishna, D. V. \& SARMA, L. V. K. V. 1969 Motion of an axisymmetric body in a rotating stratified fluid confined between two parallel planes. J. Fluid Mech. 38, 833-842.

LARSEN, L. H. 1969 Oscillations of a neutrally buoyant sphere in a stratified fluid. Deep Sea Res. $16,587-603$.

Lighthill, M. J. 1958 Introduction to Fourier Analysis and Generalised Functions. Cambridge University Press.

Lighthill, M. J. 1960 Studies on magneto-hydrodynamic waves and other anisotropic wave motions. Phil. Trans. R. Soc. Lond. A 252, 397-430.

Lighthill, M. J. 1965 Group velocity. J. Inst. Maths Applics 1, 1-28.

LighthiLl, M. J. 1967 On waves generated in dispersive systems by travelling forcing effects, with applications to the dynamics of rotating fluids. J. Fluid Mech. 27, 725-752.

Lighthill, M. J. 1978 Waves in Fluids. Cambridge University Press.

LIU, C. H. \& YEH, K. C. 1971 Excitation of acoustic-gravity waves in an isothermal atmosphere. Tellus 23, 150-163.

Mclaren, T. I., Pierce, A. D., Fohl, T. \& Murphy, B. L. 1973 An investigation of internal gravity waves generated by a buoyantly rising fluid in a stratified medium. J. Fluid Mech. 57, $229-240$.

MiLes, J. W. 1971 Internal waves generated by a horizontally moving source. Geophys. Fluid Dyn. 2, 63-87.

Miropol'skir, YU. Z. 1978 Self-similar solutions of the Cauchy problem for internal waves in an unbounded fluid. Izv. Atmos. Ocean. Phys. 14, 673-679.

Moore, D. W. \& SpIEger, E. A. 1964 The generation and propagation of waves in a compressible atmosphere. Astrophys. J. 139, 48-71.

Morgan, G. W. 1953 Remarks on the problem of slow motions in a rotating fluid. Proc. Camb. Phil. Soc. 49, 362-364.

Morse, P. M. \& Feshbach, H. 1953 Methods of Theoretical Physics. Part I. McGraw-Hill.

MowbraY, D. E. \& RARITY, B. S. H. 1967 A theoretical and experimental investigation of the phase configuration of internal waves of small amplitude in a density stratified liquid. J. Fluid Mech. 28, 1-16.

Pierce, A. D. 1963 Propagation of acoustic-gravity waves from a small source above the ground in an isothermal atmosphere. J. Acoust. Soc. Am. 35, 1798-1807.

PIerce, A. D. 1981 Acoustics. An Introduction to its Physical Principles and Applications. MeGrawHill.

Ramachandra Rao, A. 1973 A note on the application of a radiation condition for a source in a rotating stratified fluid. J. Fluid Mech. 58, 161-164.

Ramachandra RaO, A. 1975 On an oscillatory point force in a rotating stratified fluid. J. Fluid Mech. 72, 353-362.

REнм, R. G. \& RADT, H.S. 1975 Internal waves generated by a translating oscillating body. J. Fluid Mech. 68, 235-258.

Row, R. V. 1967 Acoustic-gravity waves in the upper atmosphere due to a nuclear detonation and an earthquake. J. Geophys. Res. 72, 1599-1610.

Sarma, L. V. K. V. \& Krishna, D. V. 1972 Oscillation of axisymmetric bodies in a stratified fluid. Zastosow. Matem. 13, 109-121.

Sarma, L. V. K. V. \& NaIdU, K. B. $1972 a$ Source in a rotating stratified fluid. Acta Mech. 13, 21-29.

Sarma, L. V. K. V. \& NaIdU, K. B. $1972 b$ Closed form solution for a point force in a rotating stratified fluid. Pure Appl. Geophys. 99, 156-168. 
Sekerzh-Zen'kovich, S. YA. 1979 A fundamental solution of the internal-wave operator. Sov. Phys. Dokl. 24, 347-349.

Sek ERzh-Zen'kovich, S. YA. 1981 Construction of the fundamental solution for the operator of internal waves. Appl. Maths Mech. 45, 192-198.

Sekerzh-Zen'kovich, S. YA. 1982 Cauchy problem for equations of internal waves. Appl. Maths Mech. 46, 758-764.

Soвolev, S. L. 1965 Sur une classe des problèmes de physique mathématique. In Simposio Internazionale Sulle Applicazioni Dell'Analisi Alla Fisica Matematica, Cagliari-Sassari, September 28-October 4 1964, Edizioni Cremonese (Roma), pp. 192-208.

Stevenson, T. N. 1973 The phase configuration of internal waves around a body moving in a density stratified fluid. J. Fluid Mech. 60, 759-767.

Stewartson, K. 1952 On the slow motion of a sphere along the axis of a rotating fluid. Proc. Camb. Phil. Soc. 48, 168-177.

STURova, I. V. 1980 Internal waves generated in an exponentially stratified fluid by an arbitrarily moving source. Fluid Dyn. 15, 378-383.

TeOdorovich, E. V. \& Gorodtsov, V.A. 1980 On some singular solutions of internal wave equations. Izv. Atmos. Ocean. Phys. 16, 551-553.

Tolstox, I. 1973 Wave Propagation. McGraw-Hill.

VoIsin, B. 1991 Rayonnement des ondes internes de gravité. Application aux corps en mouvement. Ph.D. thesis, Université Pierre et Marie Curie.

Watson, G. N. 1966 A Treatise on the Theory of Bessel Functions (2nd edn). Cambridge University Press.

Zavol'skiI, N. A. \& Zaitsev, A. A. 1984 Development of internal waves generated by a concentrated pulse source in an infinite uniformly stratified fluid. J. Appl. Mech. Tech. Phys. $25,862-867$. 